\title{
Convergence and Divergence of Higher-Order Hermite or Hermite-Fejér Interpolation Polynomials with Exponential-Type Weights
}

\author{
Hee Sun Jung, ${ }^{1}$ Gou Nakamura, ${ }^{2}$ \\ Ryozi Sakai, ${ }^{3}$ and Noriaki Suzuki ${ }^{3}$ \\ ${ }^{1}$ Department of Mathematics Education, Sungkyunkwan University, \\ Seoul 110-745, Republic of Korea \\ 2 Science Division, Center for General Education, Aichi Institute of Technology, \\ Yakusa-cho, Toyota 470-0392, Japan \\ ${ }^{3}$ Department of Mathematics, Meijo University, Nagoya 468-8502, Japan
}

Correspondence should be addressed to Hee Sun Jung, hsun90@skku.edu

Received 30 December 2011; Accepted 26 February 2012

Academic Editors: B. Ricceri and W. Yu

Copyright (C 2012 Hee Sun Jung et al. This is an open access article distributed under the Creative Commons Attribution License, which permits unrestricted use, distribution, and reproduction in any medium, provided the original work is properly cited.

Let $\mathbb{R}=(-\infty, \infty)$, and let $w_{\rho}(x)=|x|^{\rho} e^{-Q(x)}$, where $\rho>-1 / 2$ and $Q \in C^{1}(\mathbb{R}): \mathbb{R} \rightarrow \mathbb{R}^{+}=[0, \infty)$ is an even function. Then we can construct the orthonormal polynomials $p_{n}\left(w_{\rho}^{2} ; x\right)$ of degree $n$ for $w_{\rho}^{2}(x)$. In this paper for an even integer $v \geq 2$ we investigate the convergence theorems with respect to the higher-order Hermite and Hermite-Fejér interpolation polynomials and related approximation process based at the zeros $\left\{x_{k, n, \rho}\right\}_{k=1}^{n}$ of $p_{n}\left(w_{\rho}^{2} ; x\right)$. Moreover, for an odd integer $v \geq 1$, we give a certain divergence theorem with respect to the higher-order Hermite-Fejer interpolation polynomials based at the zeros $\left\{x_{k, n, \rho}\right\}_{k=1}^{n}$ of $p_{n}\left(w_{\rho}^{2} ; x\right)$.

\section{Introduction}

Let $\mathbb{R}=(-\infty, \infty)$, and let $Q \in C^{1}(\mathbb{R}): \mathbb{R} \rightarrow \mathbb{R}^{+}=[0, \infty)$ be an even function. Consider the weight $w(x)=\exp (-Q(x))$, and define, for $\rho>-1 / 2$,

$$
w_{\rho}(x):=|x|^{\rho} w(x), \quad x \in \mathbb{R} .
$$

Suppose that $\int_{0}^{\infty} x^{n} w_{\rho}(x) d x<\infty$, for all $n=0,1,2, \ldots$ Then we can construct the orthonormal 
polynomials $p_{n, \rho}(x)=p_{n}\left(w_{\rho}^{2} ; x\right)$ of degree $n$ for $w_{\rho}^{2}(x)$; that is,

$$
\int_{-\infty}^{\infty} p_{n, \rho}(x) p_{m, \rho}(x) w_{\rho}^{2}(x) d x=\delta_{m, n} \quad(\text { Kronecker delta) }
$$

We write $p_{n, \rho}(x)$ by

$$
p_{n, \rho}(x)=\gamma_{n} x^{n}+\cdots, \quad \gamma_{n}=\gamma_{n, \rho}>0,
$$

and denote the zeros of $p_{n, \rho}(x)$ by

$$
-\infty<x_{n, n, \rho}<x_{n-1, n, \rho}<\cdots<x_{2, n, \rho}<x_{1, n, \rho}<\infty
$$

Let $p_{n}$ denote the class of polynomials with degree at most $n$. For $f \in C(\mathbb{R})$ we define the higher-order Hermite-Fejer interpolation polynomial $L_{n}(v, f ; x) \in p_{v n-1}$ based at the zeros $\left\{x_{k, n, \rho}\right\}_{k=1}^{n}$ as follows:

$$
L_{n}^{(i)}\left(v, f ; x_{k, n, \rho}\right)=\delta_{0, i} f\left(x_{k, n, \rho}\right) \quad \text { for } k=1,2, \ldots, n, i=0,1, \ldots, v-1
$$

We note that $L_{n}(1, f ; x)$ is the Lagrange interpolation polynomial, $L_{n}(2, f ; x)$ is the ordinary Hermite-Fejér interpolation polynomial, and $L_{n}(4, f ; x)$ is the Krylov-Stayermann polynomial. For the general cases Kanjin and Sakai $[1,2]$ started to investigate the so-called Freud-type weights. The fundamental polynomials $h_{k, n, \rho}(v ; x) \in D_{v n-1}$ for the higher-order Hermite-Fejér interpolation polynomial $L_{n}(v, f ; x)$ are defined as follows:

$$
\begin{gathered}
h_{k, n, \rho}(v ; x)=l_{k, n, \rho}^{v}(x) \sum_{i=0}^{v-1} e_{i}(v, k, n)\left(x-x_{k, n, \rho}\right)^{i}, \\
l_{k, n, \rho}(x)=\frac{p_{n}\left(w_{\rho}^{2} ; x\right)}{\left(x-x_{k, n, \rho}\right) p_{n}^{\prime}\left(w_{\rho}^{2} ; x_{k, n, \rho}\right)}, \\
h_{k, n, \rho}\left(v ; x_{p, n, \rho}\right)=\delta_{k, p}, \quad h_{k, n, \rho}^{(i)}\left(v ; x_{p, n, \rho}\right)=0, \\
k, p=1,2, \ldots, n, \quad i=1,2, \ldots, v-1 .
\end{gathered}
$$

Using them, we can write

$$
L_{n}(v, f ; x)=\sum_{k=1}^{n} f\left(x_{k, n, \rho}\right) h_{k, n, \rho}(v ; x)
$$


Furthermore, we extend the operator $L_{n}(v, f ; x)$. Let $l$ be a nonnegative integer, and let $v-1 \geqslant l$. For $f \in C^{l}(\mathbb{R})$ we define the $(l, v)$-order Hermite-Fejér interpolation polynomials $L_{n}(l, v, f ; x) \in \mathcal{P}_{v n-1}$ as follows: for each $k=1,2, \ldots, n$,

$$
\begin{gathered}
L_{n}\left(l, v, f ; x_{k, n, \rho}\right)=f\left(x_{k, n, \rho}\right), \\
L_{n}^{(j)}\left(l, v, f ; x_{k, n, \rho}\right)=f^{(j)}\left(x_{k, n, \rho}\right), \quad j=1,2, \ldots, l, \\
L_{n}^{(j)}\left(l, v, f ; x_{k, n, \rho}\right)=0, \quad j=l+1, l+2, \ldots, v-1 .
\end{gathered}
$$

Especially, $L_{n}(0, v, f ; x)$ is equal to $L_{n}(v, f ; x)$, and for each $P \in p_{v n-1}$ we see $L_{n}(v-1, v, P ; x)=$ $P(x)$; that is, for $f \in C^{(v-1)}(\mathbb{R}), L_{n}(v-1, v, f ; x)$ is the Hermite interpolation polynomial. The fundamental polynomials $h_{s, k, n, \rho}(l, v ; x) \in \mathcal{D}_{v n-1}, k=1,2, \ldots, n$, of $L_{n}(l, v, f ; x)$ are defined by

$$
\begin{gathered}
h_{s, k, n, \rho}(l, v ; x)=l_{k, n, \rho}^{v}(x) \sum_{i=s}^{v-1} e_{s, i}(v, k, n)\left(x-x_{k, n, \rho}\right)^{i}, \\
h_{s, k, n, \rho}^{(j)}\left(l, v ; x_{p, n, \rho}\right)=\delta_{s, j} \delta_{k, p}, \quad j, s=0,1, \ldots, v-1, p=1,2, \ldots, n .
\end{gathered}
$$

Then we have

$$
L_{n}(l, v, f ; x)=\sum_{k=1}^{n} \sum_{s=0}^{l} f^{(s)}\left(x_{k, n, \rho}\right) h_{s, k, n, \rho}(l, v ; x)
$$

For the ordinary Hermite and Hermite-Fejer interpolation polynomial $L_{n}(1,2, f ; x)$, $L_{n}(2, f ; x)$ and the related approximation process, Lubinsky [3] gave some interesting convergence theorems.

Our purpose in this paper is to study $L_{n}(v, f ; x)$ and $L_{n}(l, v, f ; x)$ as certain analogies of the Lubinsky theorems in [3] and the related approximation process for the exponentialtype weights. Kasuga and Sakai [4-8] investigated the convergence and divergence theorems for the Freud-type weights. Then for an even integer $v \geq 2$ we give the convergence theorems for them; moreover, for an odd integer $v \geqslant 1$, we obtain a certain divergence theorem with respect to $L_{n}(v, f ; x)$. In Section 1 , we give the preliminaries for these studies, and in Section 2 we write some preliminary description. In Section 3 we report our theorems with some lemmas, and in Section 4 we prove the theorems. Finally, in Section 5, for an odd integer $v \geqslant 1$ we obtain a certain divergence theorem with respect to $L_{n}(v, f ; x)$.

In what follows we abbreviate several notations as $x_{k, n}:=x_{k, n, \rho}, h_{k n}(x):=h_{k, n, \rho}(v, x)$, $l_{k n}(x):=l_{k, n, \rho}(x), h_{s k n}(x):=h_{s, k, n, \rho}(v, x)$ and $p_{n}(x):=p_{n, \rho}(x)$ if there is no confusion. For arbitrary nonzero real-valued functions $f(x)$ and $g(x)$, we write $f(x) \sim g(x)$ if there exist constants $C_{1}, C_{2}>0$ independent of $x$ such that $C_{1} g(x) \leqslant f(x) \leqslant C_{2} g(x)$ for all $x$. For arbitrary positive sequences $\left\{c_{n}\right\}_{n=1}^{\infty}$ and $\left\{d_{n}\right\}_{=1}^{\infty}$, we define $c_{n} \sim d_{n}$ similarly.

Throughout this paper $C, C_{1}, C_{2}, \ldots$ denote positive constants independent of $n, x, t$, or polynomials $P_{n}(x)$, and the same symbol does not necessarily denote the same constant in different occurrences. 


\section{Preliminaries}

A function $f: \mathbb{R}^{+} \rightarrow \mathbb{R}^{+}$is said to be quasi-increasing if there exists $C>0$ such that $f(x) \leqslant$ $C f(y)$ for $0<x<y$.

Definition 2.1 (see [9]). Let $Q: \mathbb{R} \rightarrow \mathbb{R}^{+}$be a continuous even function satisfying the following properties.

(a) $Q^{\prime}(x)$ is continuous in $\mathbb{R}$ and $Q(0)=0$.

(b) $Q^{\prime \prime}(x)$ exists and is positive in $\mathbb{R} \backslash\{0\}$.

(c) $\lim _{x \rightarrow \infty} Q(x)=\infty$.

(d) The function

$$
T(x):=\frac{x Q^{\prime}(x)}{Q(x)}, \quad x \neq 0,
$$

is quasi-increasing in $(0, \infty)$, with $T(x) \geqslant \Lambda>1$ for $x \in \mathbb{R}^{+} \backslash\{0\}$.

(e) There exists $C_{1}>0$ such that

$$
\frac{Q^{\prime \prime}(x)}{\left|Q^{\prime}(x)\right|} \leqslant C_{1} \frac{\left|Q^{\prime}(x)\right|}{Q(x)}, \quad \text { a.e. } x \in \mathbb{R} \backslash\{0\}
$$

Then we say that $w=\exp (-Q)$ is in the class $\mathcal{F}\left(C^{2}\right)$. Besides, if there exist a compact subinterval $J(\ni 0)$ of $\mathbb{R}$ and $C_{2}>0$ such that

$$
\frac{Q^{\prime \prime}(x)}{\left|Q^{\prime}(x)\right|} \geqslant C_{2} \frac{\left|Q^{\prime}(x)\right|}{Q(x)}, \quad \text { a.e. } x \in \mathbb{R} \backslash J
$$

then we say that $w=\exp (-Q)$ is in the class $\mathcal{F}\left(C^{2}+\right)$.

Example 2.2. We present some typical examples of $Q(x)$ satisfying $w=\exp (-Q) \in \mathcal{F}\left(C^{2}+\right)$.

(1) If a continuous exponential $Q(x)$ satisfies

$$
1<\Lambda_{1} \leqslant \frac{\left(x Q^{\prime}(x)\right)^{\prime}}{Q^{\prime}(x)} \leqslant \Lambda_{2}
$$

with some constants $\Lambda_{i}, i=1,2$, then $w(x)=\exp (-Q(x))$ is called a Freud weight. The class $\mathcal{F}\left(C^{2}+\right)$ contains the Freud weights.

(2) (See [9]) For $\alpha>1$ and a nonnegative integer $r$, we put

$$
Q(x)=Q_{r, \alpha}(x):=\exp _{r}\left(|x|^{\alpha}\right)-\exp _{r}(0),
$$


where, for $r \geqslant 1$,

$$
\exp _{r}(x):=\exp (\exp (\exp (\cdots \exp x) \cdots)) \quad(r \text {-times })
$$

and $\exp _{0}(x):=x$.

(3) (See [10]) For $m \geqslant 0, \alpha \geqslant 0$ with $\alpha+m>1$, we put

$$
Q(x)=Q_{r, \alpha, m}(x):=|x|^{m}\left\{\exp _{r}\left(|x|^{\alpha}\right)-\alpha^{*} \exp _{r}(0)\right\},
$$

where for $r>0$ we suppose $\alpha^{*}=0$ if $\alpha=0 ; \alpha^{*}=1$ otherwise. For $r=0$ we suppose $m>1$ and $\alpha=0$.

(4) (See [10]) For $\alpha>1$, we put

$$
Q(x)=Q_{\alpha}(x):=(1+|x|)^{|x|^{\alpha}}-1
$$

For $x>0$, we define the Mhaskar-Rakhmanov-Saff number $a_{x}$ by the equation

$$
x=\frac{2}{\pi} \int_{0}^{1} \frac{a_{x} u Q^{\prime}\left(a_{x} u\right)}{\left(1-u^{2}\right)^{1 / 2}} d u
$$

We have the following estimates for the coefficients $e_{s, i}(v, k, n)\left(e_{i}(v, k, n):=e_{0, i}(v, k, n)\right)$ in (1.6) or (1.9).

Lemma 2.3 (see [11, Theorem 2.6]). Let $w(x)=\exp (-Q(x)) \in \mathcal{F}\left(C^{2}+\right)$. For each $k=1,2, \ldots, n$ and $s=0,1, \ldots, v-1$, we have $e_{0,0}(v, k, n)=e_{0}(v, k, n)=1$,

$$
\left|e_{s, i}(v, k, n)\right| \leqslant C\left(\frac{n}{\sqrt{a_{2 n}^{2}-x_{k, n}^{2}}}\right)^{i-s}, \quad s=1,2, \ldots, v-1, i=s, s+1, \ldots, v-1
$$

If we consider the higher-order Hermite-Fejér interpolation polynomial $L_{n}(v, f ; x)$ on a certain finite interval, then we can see a remarkable difference between the parity of $v$, for example, the Lagrange interpolation polynomial $L_{n}(1, f ; x)$ and the ordinary Hermite-Fejér interpolation polynomial $L_{n}(2, f ; x)([12-17])$. Also, we can see a similar phenomenon in the case of the infinite intervals $([1,2,4-8])$. To describe these aspects, however, we need a further strengthened definition for $v \geqslant 2$ than Definition 2.1.

Definition 2.4. Let $w(x)=\exp (-Q(x)) \in \mp\left(C^{2}+\right)$, and let $v \geqslant 1$ be an integer. Assume that $Q(x)$ is a $\mathcal{v}$-times continuously differentiable function on $\mathbb{R}$ and satisfies the following.

(a) $Q^{(v+1)}(x)$ exists and $Q^{(i)}(x), 0 \leqslant i \leqslant v+1$, are positive for $x>0$. 
(b) There exist constants $C_{i}>0$ such that

$$
\left|Q^{(i+1)}(x)\right| \leqslant C_{i}\left|Q^{(i)}(x)\right| \frac{\left|Q^{\prime}(x)\right|}{Q(x)}, \quad x \in \mathbb{R} \backslash\{0\}, \quad i=1,2, \ldots, v
$$

(c) There exist $0 \leqslant \delta<1$ and $c_{1}>0$ such that

$$
Q^{(v+1)}(x) \leqslant C\left(\frac{1}{x}\right)^{\delta}, \quad x \in\left(0, c_{1}\right]
$$

Then we say that $w(x)=\exp (-Q(x))$ is in the class $\mathscr{F}_{v}\left(C^{2}+\right)$.

(d) Suppose one of the following.

$(\mathrm{d}-1) Q^{\prime}(x) / Q(x)$ is quasi-increasing on a certain positive interval $\left[c_{2}, \infty\right)$.

$(\mathrm{d}-2) Q^{(v+1)}(x)$ is nondecreasing on a certain positive interval $\left[c_{2}, \infty\right)$.

(d-3) There exist constants $C>0$ and $0 \leqslant \delta<1$ such that $Q^{(v+1)}(x) \leqslant C(1 / x)^{\delta}$ on $(0, \infty)$.

Then one says that $w(x)=\exp (-Q(x))$ is in the class $\tilde{\mathscr{F}}_{v}\left(C^{2}+\right)$.

Example 2.5 (cf. [10, Theorem 3.1]). Let $v$ be a positive integer, and let $Q_{r, \alpha, m}$ be defined in (2.7).

(1) Let $m$ and $\alpha$ be nonnegative even integers with $m+\alpha>1$. Then $w(x)=$ $\exp \left(-Q_{r, \alpha, m}\right) \in \mathcal{F}_{v}\left(C^{2}+\right)$.

(a) If $r>0$, then we see that $Q_{r, \alpha, m}^{\prime}(x) / Q_{r, \alpha, m}(x)$ is quasi-increasing on a certain positive interval $\left(c_{1}, \infty\right)$ and $Q_{r, 0, m}(x)$ is nondecreasing on $(0, \infty)$.

(b) If $r=0$, then we see that $Q_{0,0, m}(x), m \geqslant 2$, is nondecreasing on $(0, \infty)$.

Hence, $w(x)=\exp \left(-Q_{r, \alpha, m}\right) \in \tilde{\mathcal{F}}_{v}\left(C^{2}+\right)$.

(2) Let $m+\alpha-v>0$. Then $w(x)=\exp \left(-Q_{r, \alpha, m}\right) \in \mathcal{F}_{v}\left(C^{2}+\right)$, and one has the following.

(c) If $r \geqslant 2$ and $\alpha>0$, then there exists a constant $c_{1}>0$ such that $Q_{r, \alpha, m}^{\prime}(x) / Q_{r, \alpha, m}(x)$ is quasi-increasing on $\left(c_{1}, \infty\right)$.

(d) Let $r=1$. If $\alpha \geqslant 1$, then there exists a constant $c_{2}>0$ such that $Q_{1, \alpha, m}^{\prime}(x) / Q_{1, \alpha, m}(x)$ is quasi-increasing on $\left(c_{2}, \infty\right)$, and, if $0<\alpha<1$, then $Q_{1, \alpha, m}^{\prime \prime}(x) / Q_{1, \alpha, m}(x)$ is quasi-decreasing on $\left(c_{2}, \infty\right)$.

(e) Let $r=1$ and $0<\alpha<1$, then $Q_{1, \alpha, m}^{(v+1)}(x)$ is nondecreasing on a certain positive interval on $\left(c_{2}, \infty\right)$.

Hence, $w(x)=\exp \left(-Q_{r, \alpha, m}\right) \in \tilde{\mathscr{F}}_{v}\left(C^{2}+\right)$.

Definition 2.6. One uses the following notation:

$$
\varphi_{u}(x)=\left\{\begin{array}{ll}
\frac{a_{u}}{u} \frac{1-|x| / a_{2 u}}{\sqrt{1-|x| / a_{u}+\delta_{u}}}, & |x| \leqslant a_{u} ; \\
\varphi_{u}\left(a_{u}\right), & a_{u}<|x|,
\end{array} \quad \delta_{u}=\left(u T\left(a_{u}\right)\right)^{-2 / 3}, u>0 .\right.
$$


Lemma 2.7 (see [18, Corollary 4.5]). Let $w_{\rho}(x)=|x|^{\rho} \exp (-Q(x)), \exp (-Q) \in \tilde{\boldsymbol{F}}_{v}\left(C^{2}+\right)$. If $x_{k, n} \neq 0$ and $\left|x_{k, n}\right| \leqslant a_{n}\left(1+\delta_{n}\right)$, then $e_{0}(v, k, n)=1$ and, for $i=1,2, \ldots, v-1$,

$$
\left|e_{i}(v, k, n)\right| \leqslant C\left\{\frac{T\left(a_{n}\right)}{a_{n}}+\left|Q^{\prime}\left(x_{k, n}\right)\right|+\frac{1}{\left|x_{k, n}\right|}\right\}^{\langle i\rangle}\left(\frac{n}{a_{2 n}-\left|x_{k, n}\right|}+\frac{T\left(a_{n}\right)}{a_{n}}\right)^{i-\langle i\rangle},
$$

where

$$
\langle i\rangle= \begin{cases}1, & i: \text { odd } \\ 0, & i: \text { even }\end{cases}
$$

For $x_{k, n}=0$ one has

$$
e_{0}(v, k, n)=1, \quad\left|e_{i}(v, k, n)\right| \leqslant C\left(\frac{n}{a_{n}}\right)^{i}, \quad i=1,2, \ldots, v-1
$$

Remark 2.8. In [19, Theorem 2.2] we see that $x_{1, n}<a_{n}$ if $n$ is large enough. Therefore Lemma 2.7 holds for all $x_{k, n}, k=1,2, \ldots, n$.

Levin and Lubinsky (see [9, Lemma 3.7]) showed that there exists $C>0$ such that for some $\varepsilon>0$ and for large enough $t$,

$$
T\left(a_{t}\right) \leqslant C t^{2-\varepsilon}
$$

In [20] we have the following estimations.

Lemma 2.9 (see [20, Theorem 1.6]). Let $w=\exp (-Q) \in \mathcal{F}\left(C^{2}+\right)$.

(1) Let $T(x)$ be unbounded. Then, for any $\eta>0$, there exists $C(\eta)>0$ such that, for $t \geqslant 1$,

$$
a_{t} \leqslant C(\eta) t^{\eta}
$$

(2) Let $\lambda:=C_{1}$ be the constant in Definition 2.1(e), that is,

$$
\frac{Q^{\prime \prime}(x)}{Q^{\prime}(x)} \leqslant \lambda \frac{Q^{\prime}(x)}{Q(x)}, \text { a.e. } x \in \mathbb{R} \backslash\{0\} \text {. }
$$

If $\lambda>1$, then there exists $C(\lambda, \eta)$ such that

$$
T\left(a_{t}\right) \leqslant C(\lambda, \eta) t^{2(\eta+\lambda-1) /(\lambda+1)}, \quad t \geqslant 1,
$$

and, if $0<\lambda \leqslant 1$, then for any $\eta>0$ there exists $C(\lambda, \eta)$ such that

$$
T\left(a_{t}\right) \leqslant C(\lambda, \eta) t^{\eta}, \quad t \geqslant 1 .
$$


Remark 2.10. (1) If $T(x)$ is bounded, then $w$ is called a Freud-type weight, and, if $T(x)$ is unbounded, then $w$ is called an Erdős-type weight.

(2) In (2.20) and (2.21), we set $0<\eta<2$ and

$$
\varepsilon= \begin{cases}2-\eta, & 0<\lambda \leqslant 1 \\ \frac{2(2-\eta)}{(\lambda+1)}, & \lambda>1\end{cases}
$$

Then (2.17) holds.

(3) If

$$
\limsup _{x \rightarrow \infty} \frac{Q(x) Q^{\prime \prime}(x)}{\left(Q^{\prime}(x)\right)^{2}} \leqslant 1
$$

then we have (2.21). Note that all the examples in Example 2.5 satisfy this inequality.

(4) For the Freud-type exponent $Q(x)=|x|^{m}, m>1$, we have

$$
T\left(a_{t}\right)=m, \quad a_{t} \sim t^{1 / m}
$$

(5) The inequality (2.18) implies

$$
0<C \leqslant \frac{n}{a_{n}}\left\{\frac{T\left(a_{n}\right)}{a_{n}}\right\}^{\nu-1} .
$$

\section{Theorems}

In the rest of this paper we assume the following for the weight $w$.

Assumption 3.1. Consider the weight $w_{\rho}(x)=|x|^{\rho} \exp (-Q(x)), \exp (-Q(x)) \in \tilde{\mathscr{F}}_{v}\left(C^{2}+\right), \rho \geqslant 0$.

(a) (cf. [20, Theorem 1.4]) If $T(x)$ is bounded, then we suppose, for $\delta$ in (2.12),

$$
a_{n} \leqslant C n^{1 /(1+v-\delta)}
$$

(b) There exist $0 \leqslant \gamma<1$ and $C(\gamma)>0$ such that

$$
T\left(a_{n}\right) \leqslant C(\gamma) n^{\gamma}
$$

here, if $T(x)$ is bounded, that is, a Freud-type weight, then we set $\gamma=0$, and if $T(x)$ is unbounded, that is, an Erdős-type weight, then we set $0<\gamma<1$. Define

$$
\varepsilon_{n}:= \begin{cases}\frac{a_{n}}{n}, & \frac{T\left(a_{n}\right)}{a_{n}}<1 \\ \frac{1}{n^{1-\gamma}}, & \frac{T\left(a_{n}\right)}{a_{n}} \geqslant 1 .\end{cases}
$$


Remark 3.2. (1) If $T(x)$ is unbounded, then (3.1) holds because of Lemma 2.9 (2.21).

(2) (3.2) holds for

$$
r=\frac{2(\eta+\lambda-1)}{\lambda+1}, \quad 0<\lambda<3
$$

(3) In (3.3) we note that $\varepsilon_{n} \log n \rightarrow 0$ as $n \rightarrow \infty$.

We shall state our theorems. Put

$$
\begin{gathered}
X_{n}(v, f ; x):=\sum_{j=1}^{n} f\left(x_{j, n, \rho}\right) l_{j, n, \rho}^{v}(x) \sum_{i=0}^{v-2} e_{i}(v, j, n)\left(x-x_{j, n, \rho}\right)^{i}, \\
Y_{n}(v, f ; x):=\sum_{j=1}^{n} f\left(x_{j, n, \rho}\right) l_{j, n, \rho}^{v}(x) e_{v-1}(v, j, n)\left(x-x_{j, n, \rho}\right)^{v-1}, \\
Z_{n}(l, v, f ; x):=\sum_{j=1}^{n} \sum_{s=1}^{l} f^{(s)}\left(x_{j, n, \rho}\right) l_{j, n, \rho}^{v}(x) \sum_{i=s}^{v-1} e_{s i}(v, j, n)\left(x-x_{j, n, \rho}\right)^{i} .
\end{gathered}
$$

Furthermore, we consider the class $G=\left\{g_{s} \in C(\mathbb{R}), s=l+1, l+2, \ldots, v-1\right\}$ and construct the following interpolation polynomial:

$$
W_{n}(l, v, G ; x):=\sum_{j=1}^{n} \sum_{s=l+1}^{\nu-1} g_{s}\left(x_{j, n, \rho}\right) l_{j, n, \rho}^{v}(x) \sum_{i=s}^{v-1} e_{s i}(v, j, n)\left(x-x_{j, n, \rho}\right)^{i} .
$$

Then we have

$$
\begin{gathered}
L_{n}(v, f ; x)=X_{n}(v, f ; x)+Y_{n}(v, f ; x), \\
L_{n}(l, v, f ; x)=L_{n}(v, f ; x)+Z_{n}(l, v, f ; x), \\
\tilde{L}_{n}(l, v, f \oplus G ; x):=L_{n}(v, f ; x)+Z_{n}(l, v, f ; x)+W_{n}(l, v, G ; x) .
\end{gathered}
$$

Define

$$
\Phi(x):=\frac{1}{(1+Q(x))^{2 / 3} T(x)}
$$

Here we note that, for some $d>0$,

$$
\Phi(x) \sim \frac{Q(x)^{1 / 3}}{x Q^{\prime}(x)}, \quad|x| \geqslant d>0 .
$$

Moreover, we define

$$
\Phi_{n}(x):=\max \left\{\delta_{n}, 1-\frac{|x|}{a_{n}}\right\}, \quad n=1,2,3, \ldots
$$


Lemma 3.3. Let $w=\exp (-Q) \in \mathcal{F}\left(C^{2}+\right)$. Let $L>0$ be fixed. Then one has the following:

(a) (See [9, Lemma 3.5(a)]) Uniformly for $t>0$,

$$
a_{L t} \sim a_{t} .
$$

(b) (See [9, Lemma 3.5(b)]) Uniformly for $t>0$,

$$
Q^{(j)}\left(a_{L t}\right) \sim Q^{(j)}\left(a_{t}\right), \quad j=0,1
$$

Moreover,

$$
T\left(a_{L t}\right) \sim T\left(a_{t}\right) .
$$

(c) (See $[9$, Lemma 3.11 (3.52)]) Uniformly for $t>0$,

$$
\left|1-\frac{a_{L t}}{a_{t}}\right| \sim \frac{1}{T\left(a_{t}\right)}
$$

(d) (See [9, Lemma 3.4 (3.17), (3.18)]) Uniformly for $t>0$, one has

$$
Q\left(a_{t}\right) \sim \frac{t}{\sqrt{T\left(a_{t}\right)}}, \quad Q^{\prime}\left(a_{t}\right) \sim \frac{t \sqrt{T\left(a_{t}\right)}}{a_{t}} .
$$

Lemma 3.4. For $x \in \mathbb{R}$, we have

$$
\Phi(x) \leqslant C \Phi_{n}(x), \quad n \geqslant 1 \text {. }
$$

Proof. Let $x=a_{u}, u \geqslant 1$. By Lemma 3.3(d) we have

$$
u \sim Q\left(a_{u}\right) \sqrt{T\left(a_{u}\right)} .
$$

So, we have

$$
\delta_{u}^{-1} \sim Q\left(a_{u}\right)^{2 / 3} T\left(a_{u}\right)=\frac{a_{u} Q^{\prime}\left(a_{u}\right)}{Q\left(a_{u}\right)^{1 / 3}}=\frac{x Q^{\prime}(x)}{Q^{1 / 3}(x)} \sim \Phi^{-1}\left(a_{u}\right)
$$


Now, if $u \leqslant n / 2$, then

$$
\begin{aligned}
1-\frac{a_{u}}{a_{n}} & \geqslant 1-\frac{a_{n / 2}}{a_{n}} \sim \frac{1}{T\left(a_{n}\right)} \quad(\text { by Lemma 3.2(c)) } \\
& \geqslant \frac{1}{\left(n T\left(a_{n}\right)\right)^{2 / 3}}=\delta_{n} \quad(\text { by }(2.17)) .
\end{aligned}
$$

So, we have

$$
\begin{aligned}
\Phi_{n}(x) & =1-\frac{a_{u}}{a_{n}} \geqslant 1-\frac{a_{u}}{a_{2 u}} \sim \frac{1}{T\left(a_{u}\right)} \quad(\text { by Lemma 3.2(c) }) \\
& \geqslant \frac{1}{\left(u T\left(a_{u}\right)\right)^{2 / 3}}=\delta_{u} \sim \Phi(x) \quad(\text { by (2.17) and (3.18)) }
\end{aligned}
$$

Let $n / 2<u<n$. Then we have

$$
\Phi_{n}(x) \geqslant \delta_{n} \sim \delta_{u} \sim \Phi(x) \quad \text { (by Lemmas 3.2(a), (2.17), and (3.18)). }
$$

Let $C(f)$ denote a positive constant depending only on $f$.

Assumption 3.5. Let $w_{\rho}(x)=|x|^{\rho} w(x)$ and $w(x)=\exp (-Q(x)) \in \mathcal{F}_{v}\left(C^{2}+\right), \rho \geqslant 0$. Suppose the following.

(A-1) Let $f \in C(\mathbb{R})$ satisfy that, for a given $0<\delta<1$,

$$
|f(x)| w^{\nu-\delta}(x)\left\{\left|Q^{\prime}(x)\right|+\frac{1}{|x|}\right\} \leqslant C(f), \quad x \in \mathbb{R}
$$

where we suppose $\lim \sup _{|x| \rightarrow 0}|f(x) / x| \leqslant C(f)$.

(A-2) Let $f \in C^{l}(\mathbb{R})$ for a certain $0<l \leqslant v-1$. Then we suppose that $f$ satisfies

$$
\left|f^{(s)}(x)\right|\left\{\Phi^{-3 / 4}(x) w_{\rho}(x)\right\}^{v} \leqslant C(f), \quad x \in \mathbb{R}, s=1,2, \ldots, l .
$$

(A-3) Let $G=\left\{g_{s} \in C(\mathbb{R}), s=l+1, l+2, \ldots, v-1\right\}$. Then we suppose that there exists a constant $C>0$ such that

$$
\left|g_{s}(x)\right|\left\{\Phi^{-3 / 4}(x) w_{\rho}(x)\right\}^{v} \leqslant C(G), \quad x \in \mathbb{R}, s=l+1, l+2, \ldots, v-1
$$


Remark 3.6. In (3.22), we have the following.

(1) $f(0)=0$ and

$$
|f(x)|\left\{\Phi^{-3 / 4}(x) w_{\rho}(x)\right\}^{\nu}\left(\left|Q^{\prime}(x)\right|+\frac{1}{|x|}\right) \leqslant C(f), \quad x \in \mathbb{R} \backslash\{0\} .
$$

(2) For some positive constant $C$, we have $\left|Q^{\prime}(x)\right|+1 /|x| \geqslant C$. Hence, from (3.22), it follows that

$$
|f(x)|\left\{\Phi^{-3 / 4}(x) w_{\rho}(x)\right\}^{\nu} \leqslant C(f), \quad x \in \mathbb{R}
$$

We have a chain of results under Assumption 3.1.

Proposition 3.7. Let $v=1,2,3, \ldots$. For $f(x)$ satisfying (3.26), one has

$$
\left\|\left\{\Phi^{3 / 4}(x) w(x)\left(|x|+\frac{a_{n}}{n}\right)^{\rho}\right\}^{v} X_{n}(v, f ; x)\right\|_{L_{\infty}(\mathbb{R})} \leqslant C(f) .
$$

Proposition 3.8. Let $v=2,4,6, \ldots$. For $f(x)$ satisfying (3.25), one has

$$
\left\|\left\{\Phi^{3 / 4}(x) w(x)\left(|x|+\frac{a_{n}}{n}\right)^{\rho}\right\}^{v} Y_{n}(v, f ; x)\right\|_{L_{\infty}(\mathbb{R})} \leqslant C(f) \varepsilon_{n} \log n,
$$

where $\varepsilon_{n}$ is defined by (3.3).

Proposition 3.9. Let $v=1,2,3, \ldots$ For $f(x)$ satisfying (3.23), one has

$$
\left\|\left\{\Phi^{3 / 4}(x) w(x)\left(|x|+\frac{a_{n}}{n}\right)^{\rho}\right\}^{v} Z_{n}(l, v, f ; x)\right\|_{L_{\infty}(\mathbb{R})} \leqslant C(f) \frac{a_{n} \log n}{n},
$$

and for $f(x)$ satisfying (3.24) one has

$$
\left\|\left\{\Phi^{3 / 4}(x) w(x)\left(|x|+\frac{a_{n}}{n}\right)^{\rho}\right\}^{\nu} W_{n}(l, v, G ; x)\right\|_{L_{\infty}(\mathbb{R})} \leqslant C(G) \frac{a_{n} \log n}{n} .
$$

Proposition 3.10. Let $v=1,2,3, \ldots$.. Let $P \in D_{m}$ be fixed. Then one has

$$
\left\|\left\{\Phi^{3 / 4}(x) w(x)\left(|x|+\frac{a_{n}}{n}\right)^{\rho}\right\}^{\nu}\left(L_{n}(\nu, P, x)-P(x)\right)\right\|_{L_{\infty}(\mathbb{R})} \longrightarrow 0 \text { as } n \longrightarrow \infty .
$$

Proposition 3.11. Let $v=2,4,6, \ldots$ Let $P \in P_{m}$ with $P(0)=0$ be fixed. Then one has

$$
\left\|\left\{\Phi^{3 / 4}(x) w(x)\left(|x|+\frac{a_{n}}{n}\right)^{\rho}\right\}^{v}\left(X_{n}(v, P ; x)-P(x)\right)\right\|_{L_{\infty}(\mathbb{R})} \longrightarrow 0 \text { as } n \longrightarrow \infty .
$$


Theorem 3.12. Let $v=2,4,6, \ldots$ For $f(x)$ satisfying (3.22), one has

$$
\left\|\left\{\Phi^{3 / 4}(x) w(x)\left(|x|+\frac{a_{n}}{n}\right)^{\rho}\right\}^{v}\left(L_{n}(v, f ; x)-f(x)\right)\right\|_{L_{\infty}(\mathbb{R})} \longrightarrow 0 \quad \text { as } n \longrightarrow \infty .
$$

Theorem 3.13. Let $v=2,4,6, \ldots$.. For $f(x)$ satisfying (3.22) and (3.23), one has

$$
\left\|\left\{\Phi^{3 / 4}(x) w(x)\left(|x|+\frac{a_{n}}{n}\right)^{\rho}\right\}^{v}\left(L_{n}(l, v, f ; x)-f(x)\right)\right\|_{L_{\infty}(\mathbb{R})} \longrightarrow 0 \quad \text { as } n \longrightarrow \infty .
$$

Corollary 3.14. Let $v=2,4,6, \ldots$. For $f(x)$ satisfying (3.22), one has

$$
\left\|\left\{\Phi^{3 / 4}(x) w(x)\left(|x|+\frac{a_{n}}{n}\right)^{\rho}\right\}^{v}\left(X_{n}(v, f ; x)-f(x)\right)\right\|_{L_{\infty}(\mathbb{R})} \longrightarrow 0 \quad \text { as } n \longrightarrow \infty .
$$

Theorem 3.15. Let $v=2,4,6, \ldots$ For $f(x)$ satisfying (3.22), (3.23) and (3.24), one has

$$
\left\|\left\{\Phi^{3 / 4}(x) w(x)\left(|x|+\frac{a_{n}}{n}\right)^{\rho}\right\}^{v}\left(\tilde{L}_{n}(l, v, f \oplus G ; x)-f(x)\right)\right\|_{L_{\infty}(\mathbb{R})} \longrightarrow 0 \quad \text { as } n \longrightarrow \infty \text {. }
$$

Define

$$
I_{n}\left[k, H_{n}(f)\right]:=\int_{-\infty}^{\infty} k(x) H_{n}(f ; x) d x
$$

where $H_{n}(f)$ equals to one of the following:

$$
L_{n}(v, f), L_{n}(l, v, f), \tilde{L}_{n}(l, v, f \oplus G), X_{n}(v, f)
$$

One also defines

$$
I[k, f]:=\int_{-\infty}^{\infty} k(x) f(x) d x
$$

Theorem 3.16. Let $v=2,4,6, \ldots$ Let $f(x)$ satisfy (3.22), (3.23) and (3.24). If

$$
\int_{-\infty}^{\infty} k(x)\left\{\Phi^{3 / 4}(x) w(x)\left(|x|+\frac{a_{n}}{n}\right)^{\rho}\right\}^{-v} d x<\infty, \quad n=1,2,3, \ldots
$$

then we have

$$
\lim _{n \rightarrow \infty} I_{n}\left[k, H_{n}(f)\right]=I[k, f]
$$


For example, we can take $k(x)$ in the following way. If $T(x)$ is unbounded, we have, for $\Delta \in \mathbb{R}$,

$$
\int_{1}^{\infty}(1+|x|)^{-\Delta} \Phi^{3 v / 4}(x) d x \leqslant \int_{1}^{\infty}(1+|x|)^{-\Delta}\left(\frac{1}{T(x) Q(x)^{2 / 3}}\right)^{3 v / 4} d x<\infty
$$

(see [10, Lemma 2.1(b)]). Then we set

$$
k(x):=(1+|x|)^{\rho v-\Delta}\left\{\Phi^{3 / 2}(x) w(x)\right\}^{v} .
$$

If $T(x)$ is bounded and $w(x)=\exp \left(-|x|^{\beta}\right), \beta>1$, then we take $\Delta$ as $v \beta / 2+\Delta>1$ so that (3.43) can hold. Then $k(x)$ defined by (3.43) satisfies (3.40).

\section{Proof of Theorems}

For constants $C, C_{1}>0$, the same symbol does not necessarily denote the same constant in different occurrences.

Lemma 4.1. One has the following. (1) (See [19, Theorem 2.3]) Let $w(x)=\exp (-Q(x)) \in \mathcal{F}\left(C^{2}\right)$ and $\rho>-1 / 2$. Then, uniformly for $n \geqslant 1$ one has

$$
\sup _{x \in \mathbb{R}}\left|p_{n, \rho}(x) w(x)\right|\left(|x|+\frac{a_{n}}{n}\right)^{\rho}\left|x^{2}-a_{n}^{2}\right|^{1 / 4} \sim 1
$$

and for $w(x)=\exp (-Q(x)) \in \mathcal{F}\left(C^{2}+\right)$,

$$
\sup _{x \in \mathbb{R}}\left|p_{n, \rho}(x) w(x)\right|\left(|x|+\frac{a_{n}}{n}\right)^{\rho} \sim a_{n}^{-1 / 2}\left(n T\left(a_{n}\right)\right)^{1 / 6} .
$$

(2) (See [19, Theorem 2.5(d)]) Let $w \in F\left(C^{2}+\right)$ and $\rho>-1 / 2$. Let $1 \leqslant j \leqslant n-1$ and $x \in\left[x_{j+1, n}, x_{j, n}\right]$. Then

$$
\left|p_{n}(x) w(x)\right|\left(|x|+\frac{a_{n}}{n}\right)^{\rho} \sim \min \left\{\left|x-x_{j, n}\right|,\left|x-x_{j+1, n}\right|\right\} \varphi_{n}\left(x_{j, n}\right)^{-1}\left|a_{n}^{2}-x_{j, n}^{2}\right|^{-1 / 4}
$$

(3) (See [19, Theorem 2.5(c)]) Let $w \in \mathcal{F}\left(C^{2}+\right)$ and $\rho>-1 / 2$. Then one has

$$
\begin{aligned}
\max _{x \in \mathbb{R}}\left|\frac{p_{n}(x) w(x)\left(|x|+a_{n} / n\right)^{\rho}}{\left(x-x_{j, n}\right) p_{n}^{\prime}\left(x_{j, n}\right) w\left(x_{j, n}\right)\left(\left|x_{j, n}\right|+a_{n} / n\right)^{\rho}}\right| \\
\quad=\max _{x \in \mathbb{R}}\left|l_{j n}(x) w(x)\left(|x|+\frac{a_{n}}{n}\right)^{\rho}\right| w^{-1}\left(x_{j, n}\right)\left(\left|x_{j, n}\right|+\frac{a_{n}}{n}\right)^{\rho} \sim 1 .
\end{aligned}
$$


(4) (See [19, Theorem 2.5(a)]) Let $w \in \mathcal{F}\left(C^{2}+\right)$ and $\rho>-1 / 2$. For $1 \leqslant j \leqslant n$ we have

$$
\left|p_{n}^{\prime} w\right|\left(x_{j, n}\right)\left(\left|x_{j, n}\right|+\frac{a_{n}}{n}\right)^{\rho} \sim \varphi_{n}^{-1}\left(x_{j, n}\right)\left|a_{n}^{2}-x_{j, n}^{2}\right|^{-1 / 4} .
$$

Lemma 4.2 (see [19, Theorem 2.2]). Let $w(x)=\exp (-Q(x)) \in \mathcal{F}\left(C^{2}+\right)$ and $\rho>-1 / 2$. For the zeros $x_{j, n}=x_{j, n, \rho}$, one has the following:

(1) For $n \geqslant 1$ and $1 \leqslant j \leqslant n-1$,

$$
\begin{gathered}
x_{j, n}-x_{j+1, n} \sim \varphi_{n}\left(x_{j, n}\right), \\
\varphi_{n}\left(x_{j, n}\right) \sim \varphi_{n}\left(x_{j+1, n}\right) \quad(\text { see }([19, \text { Lemma A.1 }(A .3)])) .
\end{gathered}
$$

(2) For the minimum positive zero $x_{[n / 2], n}([n / 2]$ is the largest integer $\leqslant n / 2)$, one has

$$
x_{[n / 2], n} \sim a_{n} n^{-1},
$$

and for large enough $n$,

$$
1-\frac{x_{1, n}}{a_{n}} \sim \delta_{n}
$$

(3) (See [19, Lemma 4.7]) $b_{n}=\gamma_{n-1} / \gamma_{n} \sim a_{n} \sim x_{1, n}$, where $\gamma_{n}$ is defined by (1.3).

Lemma 4.3. Let $w \in \mathcal{F}\left(C^{2}+\right)$. Then there exist $C_{1}, C_{2}>0$ such that

$$
\begin{aligned}
\sup _{x \in \mathbb{R}}\left|p_{n, \rho}(x) w(x)\right|\left(|x|+\frac{a_{n}}{n}\right)^{\rho} \Phi^{1 / 4}(x) & \leqslant C_{1} \sup _{x \in \mathbb{R}}\left|p_{n, \rho}(x) w(x)\right|\left(|x|+\frac{a_{n}}{n}\right)^{\rho} \Phi_{n}^{1 / 4}(x) \\
& \leqslant C_{2} a_{n}^{-1 / 2} .
\end{aligned}
$$

Proof. The first inequality follows from Lemma 3.4. We show the second inequality. Noting (4.8), from Lemma 4.1 (4.1) we have

$$
\begin{aligned}
C & \geqslant \sup _{|x| \leqslant x_{1, n}}\left|p_{n, \rho}(x) w(x)\right|\left(|x|+\frac{a_{n}}{n}\right)^{\rho}\left|x^{2}-a_{n}^{2}\right|^{1 / 4} \\
& \sim \sup _{|x| \leqslant x_{1, n}}\left|p_{n, \rho}(x) w(x)\right|\left(|x|+\frac{a_{n}}{n}\right)^{\rho} a_{n}^{1 / 2} \Phi_{n}^{1 / 4}(x) .
\end{aligned}
$$


From (4.2) we see that

$$
\begin{aligned}
a_{n}^{-1 / 2} \geqslant & C \sup _{|x|>x_{1, n}}\left|p_{n, \rho}(x) w(x)\right|\left(|x|+\frac{a_{n}}{n}\right)^{\rho} \delta_{n}^{1 / 4} \\
& \sim C \sup _{|x|>x_{1, n}}\left|p_{n, \rho}(x) w(x)\right|\left(|x|+\frac{a_{n}}{n}\right)^{\rho} \Phi_{n}^{1 / 4}(x) .
\end{aligned}
$$

Therefore we have the result.

Proof of Proposition 3.7. We recall the definition of $X_{n}(v, f ; x)$ :

$$
\begin{aligned}
X_{n}(\nu, f ; x) & =\sum_{j=1}^{n} f\left(x_{j, n}\right) l_{j n}^{v}(x) \sum_{i=0}^{v-2} e_{i}(v, j, n)\left(x-x_{j, n}\right)^{i} \\
& =\sum_{i=0}^{v-2} \sum_{j=1}^{n} f\left(x_{j, n}\right) l_{j n}^{v}(x)\left(x-x_{j, n}\right)^{i} e_{i}(v, j, n) .
\end{aligned}
$$

Using Lemma 2.3, we may estimate, for $i=0,1, \ldots, v-2$ and $1 \leqslant j \leqslant n$,

$$
A_{i, j}(x):=\left(\Phi^{3 / 4}(x) w(x)\left(|x|+\frac{a_{n}}{n}\right)^{\rho}\right)^{v}\left|f\left(x_{j, n}\right)\right|\left|l_{j n}(x)\right|^{v}\left|x-x_{j, n}\right|^{i}\left(\frac{n}{\sqrt{a_{2 n}^{2}-x_{j, n}^{2}}}\right)^{i} .
$$

Let $\left(x_{m+1, n}+x_{m, n}\right) / 2<x \leqslant x_{m, n}$ or $x_{m, n} \leqslant x<\left(x_{m-1, n}+x_{m, n}\right) / 2$. For simplicity, we assume $x>0$, and let $x_{0, n}:=x_{1, n}+\delta \varphi_{n}\left(x_{1, n}\right)$ for a fixed $\delta>0$ small enough. Then we can assume that there exists a constant $c>0$ such that

$$
x_{0, n}:=x_{1, n}+\delta \varphi_{n}\left(x_{1, n}\right)<a_{n}-c \delta_{n}
$$

Assume that $x<x_{0, n}$. Then we first estimate $A_{i, m}(x)$. By Lemma 4.1(3) and the definition of $\varphi_{n}(x)$, we have

$$
\begin{gathered}
\left(\Phi^{3 / 4}(x) w(x)\left(|x|+\frac{a_{n}}{n}\right)^{\rho}\right)^{v}\left|l_{m n}(x)\right|^{v} \leqslant C \Phi^{3 v / 4}(x)\left(w\left(x_{m, n}\right)\left(\left|x_{m, n}\right|+\frac{a_{n}}{n}\right)^{\rho}\right)^{v} \\
\left|x-x_{m, n}\right|^{i}\left(\frac{n}{\sqrt{a_{2 n}^{2}-x_{m, n}^{2}}}\right)^{i} \leqslant C\left(\frac{n \varphi_{n}\left(x_{m, n}\right)}{\sqrt{a_{2 n}^{2}-x_{m, n}^{2}}}\right)^{i} \leqslant C\left(\frac{\sqrt{1-\left|x_{m, n}\right| / a_{2 n}}}{\sqrt{1-\left|x_{m, n}\right| / a_{n}}}\right)^{i}
\end{gathered}
$$

and by Remark 3.6

$$
\left|f\left(x_{m, n}\right)\right| \leqslant C(f) \Phi^{3 v / 4}\left(x_{m, n}\right)\left(w\left(x_{m, n}\right)\left(\left|x_{m, n}\right|+\frac{a_{n}}{n}\right)^{\rho}\right)^{-v}
$$


Therefore, we have

$$
\begin{aligned}
A_{i, m}(x) & \leqslant C(f) \Phi^{3 v / 4}(x) \Phi^{3 v / 4}\left(x_{m, n}\right)\left(\frac{\sqrt{1-\left|x_{m, n}\right| / a_{2 n}}}{\sqrt{1-\left|x_{m, n}\right| / a_{n}}}\right)^{i} \\
& =C(f) \Phi^{3 v / 4}(x)\left(\max \left\{1-\frac{\left|x_{m, n}\right|}{a_{n}}, \delta_{n}\right\}\right)^{3 v / 4}\left(\frac{\sqrt{1-\left|x_{m, n}\right| / a_{2 n}}}{\sqrt{1-\left|x_{m, n}\right| / a_{n}}}\right)^{i} \\
& =C(f) \Phi^{3 v / 4}(x)\left(1-\frac{\left|x_{m, n}\right|}{a_{n}}\right)^{3 v / 4-i / 2}\left(\sqrt{1-\frac{\left|x_{m, n}\right|}{a_{2 n}}}\right)^{i} \\
& \leqslant C(f) .
\end{aligned}
$$

Next, we estimate $\sum_{1 \leqslant j \leqslant n, j \neq m} A_{i, j}(x)$. For $1 \leqslant j \leqslant n, j \neq m$, we have, by Lemma 4.3 and Lemma 4.1(4),

$$
\begin{aligned}
& \left(\Phi^{3 / 4}(x) w(x)\left(|x|+a_{n} / n\right)^{\rho}\right)^{v}\left|l_{j n}(x)\right|^{v}\left|x-x_{j, n}\right|^{i} \\
& \quad=\Phi^{v / 2}(x)\left|\frac{p_{n}(x) \Phi^{1 / 4}(x) w(x)\left(|x|+a_{n} / n\right)^{\rho}}{p_{n}^{\prime}\left(x_{j, n}\right) w\left(x_{j, n}\right)\left(\left|x_{j, n}\right|+a_{n} / n\right)^{\rho}}\right|^{v} \frac{\left(w\left(x_{j, n}\right)\left(\left|x_{j, n}\right|+a_{n} / n\right)^{\rho}\right)^{v}}{\left|x-x_{j, n}\right|^{v-i}} \\
& \quad=\Phi^{v / 2}(x) a_{n}^{-v / 2} \varphi_{n}^{v}\left(x_{j, n}\right)\left|a_{n}^{2}-x_{j, n}^{2}\right|^{v / 4} \frac{\left(w\left(x_{j, n}\right)\left(\left|x_{j, n}\right|+a_{n} / n\right)^{\rho}\right)^{v}}{\left|x-x_{j, n}\right|^{v-i}}
\end{aligned}
$$

Then, since we know from Remark 3.6 that

$$
\left|f\left(x_{j, n}\right)\right| \leqslant C(f) \Phi^{3 v / 4}\left(x_{j, n}\right)\left(w\left(x_{j, n}\right)\left(\left|x_{j, n}\right|+\frac{a_{n}}{n}\right)^{\rho}\right)^{-v}
$$

we have, by Lemma 3.4,

$$
\begin{aligned}
\sum_{j \neq m} A_{i, j}(x) \leqslant & C(f) \sum_{j \neq m} \Phi_{n}^{v / 2}(x) \Phi_{n}^{3 v / 4}\left(x_{j, n}\right) \varphi_{n}^{v}\left(x_{j, n}\right) \\
& \times\left(1-\frac{\left|x_{j, n}\right|}{a_{n}}\right)^{v / 4}\left(\frac{1}{\left|x-x_{j, n}\right|}\right)^{v-i}\left(\frac{n}{\sqrt{a_{2 n}^{2}-x_{j, n}^{2}}}\right)^{i} .
\end{aligned}
$$

By the definition of $\varphi_{n}(x)$ and by Lemma 3.3(a) $a_{2 n} \sim a_{n}$, we have

$$
\varphi_{n}^{v}\left(x_{j, n}\right)\left(\frac{n}{\sqrt{a_{2 n}^{2}-x_{j, n}^{2}}}\right)^{i} \leqslant C\left(\frac{a_{n}}{n}\right)^{v-i}\left(1-\frac{\left|x_{j, n}\right|}{a_{2 n}}\right)^{v-i / 2}\left(1-\frac{\left|x_{j, n}\right|}{a_{n}}\right)^{-v / 2} .
$$


By Lemma 4.2(1), we have

$$
\begin{aligned}
\frac{1}{\left|x-x_{j, n}\right|} & \leqslant C \frac{1}{\sum_{j \leqslant s \leqslant m+1 \text { or } m-1 \leqslant s \leqslant j} \varphi_{n}\left(x_{s, n}\right)} \\
& \leqslant C \frac{n / a_{n}}{\sum_{j \leqslant s \leqslant m+1 \text { or } m-1 \leqslant s \leqslant j}\left(1-\left|x_{s, n}\right| / a_{2 n}\right) /\left(1-\left|x_{s, n}\right| / a_{n}+\delta_{n}\right)^{1 / 2}} \\
& \leqslant C \frac{n / a_{n}}{\sum_{j \leqslant s \leqslant m+1 \text { or } m-1 \leqslant s \leqslant j}\left(1-\left|x_{s, n}\right| / a_{n}\right) /\left(1-\left|x_{s, n}\right| / a_{n}+\delta_{n}\right)^{1 / 2}} \\
& \leqslant C \frac{n / a_{n}}{|m-j|\left(1-\max \left\{|x|,\left|x_{j, n}\right|\right\} / a_{n}\right)^{1 / 2}} .
\end{aligned}
$$

Here, we note from (4.14) and (4.8) that

$$
\Phi_{n}(x) \sim\left(1-\frac{|x|}{a_{n}}\right), \quad \Phi_{n}\left(x_{j, n}\right) \sim\left(1-\frac{\left|x_{j, n}\right|}{a_{n}}\right) .
$$

Thus, we have, for $j \neq m$,

$$
\begin{aligned}
\Phi_{n}^{v / 2}(x) & \Phi_{n}^{3 v / 4}\left(x_{j, n}\right) \varphi_{n}^{v}\left(x_{j, n}\right)\left(1-\frac{\left|x_{j, n}\right|}{a_{n}}\right)^{v / 4}\left(\frac{1}{\left|x-x_{j, n}\right|}\right)^{v-i}\left(\frac{n}{\sqrt{a_{2 n}^{2}-x_{j, n}^{2}}}\right)^{i} \\
\leqslant & C\left(1-\frac{|x|}{a_{n}}\right)^{v / 2}\left(1-\frac{\left|x_{j, n}\right|}{a_{n}}\right)^{v / 2}\left(1-\frac{\left|x_{j, n}\right|}{a_{2 n}}\right)^{v-i / 2} \\
& \times\left(\frac{1}{|m-j|\left(1-\max \left\{|x|,\left|x_{j, n}\right|\right\} / a_{n}\right)^{1 / 2}}\right)^{v-i} \\
\leqslant & C\left(\frac{1}{|m-j|}\right)^{v-i} .
\end{aligned}
$$

Therefore, we have

$$
\sum_{\substack{1 \leqslant j \leqslant n, j \neq m}} A_{i, j}(x) \leqslant C(f) \sum_{j \neq m}\left(\frac{1}{|m-j|}\right)^{v-i} \leqslant C(f),
$$

because of $v-i \geqslant 2$.

Remark 4.4. If we consider the estimate of $\sum_{i=1}^{n} A_{i, v-1}(x)$ with (4.13), then we obtain

$$
\sum_{i=1}^{n} A_{i, v-1}(x) \leqslant C(f) \log n
$$


We continue the proof Proposition 3.7. We need to estimate $\sum_{j=1}^{n} A_{i, j}(x)$ for $|x|>x_{0, n}$. Now, suppose $x>x_{0, n}$. Then similarly to (4.23), we have

$$
\frac{1}{\left|x_{0, n}-x_{j, n}\right|} \leqslant C \frac{1}{\sum_{1 \leqslant s \leqslant j} \varphi_{n}\left(x_{s, n}\right)} \leqslant C \frac{n / a_{n}}{j\left(1-x_{0, n} / a_{n}\right)^{1 / 2}} \leqslant C \frac{1}{j \delta_{n}^{1 / 2}} \frac{n}{a_{n}} .
$$

Similarly to (4.21), we have using (4.22)

$$
\begin{aligned}
\sum A_{i, j}(x) \leqslant & C(f) \sum_{j} \Phi^{v / 2}(x) \Phi_{n}^{3 v / 4}\left(x_{j, n}\right) \varphi_{n}^{v}\left(x_{j, n}\right) \\
& \times\left(1-\frac{\left|x_{j, n}\right|}{a_{n}}\right)^{v / 4}\left(\frac{1}{\left|x_{0, n}-x_{j, n}\right|}\right)^{v-i}\left(\frac{n}{\sqrt{a_{2 n}^{2}-x_{j, n}^{2}}}\right)^{i} \\
\leqslant & C(f) \sum_{j} \Phi_{n}^{v / 2}(x)\left(1-\frac{\left|x_{j, n}\right|}{a_{n}}\right)^{v / 2}\left(1-\frac{\left|x_{j, n}\right|}{a_{2 n}}\right)^{v-i / 2}\left(\frac{1}{j \delta_{n}^{1 / 2}}\right)^{v-i} \\
\leqslant & C(f) \sum_{j} \frac{1}{j^{v-i}} C(f),
\end{aligned}
$$

because of $v-i \geqslant 2$ and $\Phi^{v / 2}(x) \delta_{n}^{-(v-i) / 2} \leqslant \delta_{n}^{v / 2} \delta_{n}^{-(v-i) / 2} \leqslant C$. Consequently we achieve the result.

Remark 4.5. The above proof implies the following: there exists a constant $C>0$ such that

$$
\sum_{j=1}^{n}\left|h_{j, n, \rho}(v ; x)\right| \leqslant \sum_{j=1}^{n}\left|l_{j n}(x)\right|^{\nu} \sum_{i=0}^{\nu-2}\left|e_{i}(v, k, n)\right|\left|x-x_{j, n}\right|^{i} \leqslant C, \quad x \in \mathbb{R} .
$$

Proof of Proposition 3.8. We use the same method to prove of Proposition 3.7. So, we let $\left(x_{m+1, n}+x_{m, n}\right) / 2<x \leqslant x_{m, n}$ or $x_{m, n} \leqslant x<\left(x_{m-1, n}+x_{m, n}\right) / 2$. For simplicity, we assume $x>0$, and let $x_{0, n}:=x_{1, n}+\delta \varphi_{n}\left(x_{1, n}\right)$ for a fixed $\delta>0$ small enough and there exists a constant $\delta_{1}>0$ such that $x_{0, n}:=x_{1, n}+\delta \varphi_{n}\left(x_{1, n}\right)<a_{n}-\delta_{1} \delta_{n}$. Assume that $x<x_{0, n}$. Since $f(0)=0$, we may leave out the term with $x_{j, n}=0$. Hence we consider only the term of $x_{j, n} \neq 0$. Noting Assumption 3.1, (3.2), and Lemma 2.7, we estimate $\sum_{j=1}^{n} B_{v-1, j}(x)$, where

$$
\begin{aligned}
B_{v-1, j}(x):= & \left(\Phi^{3 / 4}(x) w(x)\left(|x|+\frac{a_{n}}{n}\right)^{\rho}\right)^{\nu} \\
& \times\left|f\left(x_{j, n}\right)\right|\left|l_{j n}(x)\right|^{\nu}\left|x-x_{j, n}\right|^{\nu-1}\left\{\frac{T\left(a_{n}\right)}{a_{n}}+\left|Q^{\prime}\left(x_{j, n}\right)\right|+\frac{1}{\left|x_{j, n}\right|}\right\}\left(\frac{n}{a_{2 n}-\left|x_{j, n}\right|}\right)^{v-2} .
\end{aligned}
$$


First we estimate $B_{v-1, m}(x)$. By Lemma 4.2, (4.16), and the definition of $\varphi_{n}(x)$, we have

$$
\begin{aligned}
\left|x-x_{m, n}\right|^{\nu-1}\left(\frac{n}{\sqrt{a_{2 n}^{2}-x_{m, n}^{2}}}\right)^{\nu-2} & \leqslant C \varphi_{n}\left(x_{m, n}\right)\left(\frac{\sqrt{1-\left|x_{m, n}\right| / a_{2 n}}}{\sqrt{1-\left|x_{m, n}\right| / a_{n}}}\right)^{\nu-2} \\
& \leqslant C \frac{a_{n}}{n}\left(1-\frac{\left|x_{m, n}\right|}{a_{2 n}}\right)^{1 / 2}\left(\frac{\sqrt{1-\left|x_{m, n}\right| / a_{2 n}}}{\sqrt{1-\left|x_{m, n}\right| / a_{n}}}\right)^{\nu-1} .
\end{aligned}
$$

Since here $\left|Q^{\prime}\left(x_{m, n}\right)\right|+1 /\left|x_{m, n}\right| \geqslant C$ for some positive constant $C$, we know that

$$
\left(\frac{T\left(a_{n}\right)}{a_{n}}+\left|Q^{\prime}\left(x_{m, n}\right)\right|+\frac{1}{\left|x_{m, n}\right|}\right) \leqslant C \max \left\{1, \frac{T\left(a_{n}\right)}{a_{n}}\right\}\left(\left|Q^{\prime}\left(x_{m, n}\right)\right|+\frac{1}{\left|x_{m, n}\right|}\right),
$$

and by (3.25)

$$
\left|f\left(x_{m, n}\right)\right| \leqslant C(f) \Phi^{3 v / 4}\left(x_{m, n}\right)\left(w\left(x_{m, n}\right)\left(\left|x_{m, n}\right|+\frac{a_{n}}{n}\right)^{\rho}\right)^{-v}\left(\left|Q^{\prime}\left(x_{m, n}\right)\right|+\frac{1}{\left|x_{m, n}\right|}\right)^{-1} .
$$

Therefore, using (3.3) and (4.15), we have

$$
\begin{aligned}
B_{v-1, m}(x) \leqslant & C(f) \max \left\{1, \frac{T\left(a_{n}\right)}{a_{n}}\right\} \Phi_{n}^{3 v / 4}(x) \Phi_{n}^{3 v / 4}\left(x_{m, n}\right) \frac{a_{n}}{n}\left(1-\frac{\left|x_{m, n}\right|}{a_{2 n}}\right)^{1 / 2} \\
& \times\left(\frac{\sqrt{1-\left|x_{m, n}\right| / a_{2 n}}}{\sqrt{1-\left|x_{m, n}\right| / a_{n}}}\right)^{v-1} \\
\leqslant & C(f) \varepsilon_{n} \Phi_{n}^{3 v / 4}(x) \Phi_{n}^{3 v / 4}\left(x_{m, n}\right)\left(1-\frac{\left|x_{m, n}\right|}{a_{2 n}}\right)^{1 / 2}\left(\frac{\sqrt{1-\left|x_{m, n}\right| / a_{2 n}}}{\sqrt{1-\left|x_{m, n}\right| / a_{n}}}\right)^{v-1} .
\end{aligned}
$$

Noting (4.24), we have

$$
B_{v-1, m}(x) \leqslant C(f) \varepsilon_{n} \Phi_{n}^{3 v / 4}(x)\left(1-\frac{\left|x_{m, n}\right|}{a_{n}}\right)^{(v+2) / 4}\left(1-\frac{\left|x_{m, n}\right|}{a_{2 n}}\right)^{v / 2} \leqslant C(f) \varepsilon_{n} .
$$

Next, we estimate $\sum_{j \neq m} B_{v-1, j}(x)$. Noting (4.19) and (4.22), we have

$$
\begin{gathered}
\left(\Phi_{n}^{3 / 4}(x) w(x)\left(|x|+\frac{a_{n}}{n}\right)^{\rho}\right)^{v}\left|l_{j n}(x)\right|^{v}\left|x-x_{j, n}\right|^{v-1} \\
=\Phi_{n}^{v / 2}(x) a_{n}^{-v / 2} \varphi_{n}^{v}\left(x_{j, n}\right)\left|a_{n}^{2}-x_{j, n}^{2}\right|^{v / 4} \frac{\left(w\left(x_{j, n}\right)\left(\left|x_{j, n}\right|+a_{n} / n\right)^{\rho}\right)^{v}}{\left|x-x_{j, n}\right|} \\
\varphi_{n}^{v}\left(x_{j, n}\right)\left(\frac{n}{\sqrt{a_{2 n}^{2}-x_{j, n}^{2}}}\right)^{v-2} \leqslant C\left(\frac{a_{n}}{n}\right)^{2}\left(1-\frac{\left|x_{j, n}\right|}{a_{n}}\right)^{-v / 2}\left(1-\frac{\left|x_{j, n}\right|}{a_{2 n}}\right)^{v / 2+1} .
\end{gathered}
$$


Then by (4.33) and (3.25) (noting (4.34)), using the notation (3.3) and (4.24), we have

$$
\begin{aligned}
B_{v-1, j}(x) \leqslant & C(f) \max \left\{1, \frac{T\left(a_{n}\right)}{a_{n}}\right\}\left(\frac{a_{n}}{n}\right)^{2} \Phi_{n}^{v / 2}(x) \Phi_{n}^{3 v / 4}\left(x_{j, n}\right) a_{n}^{-v / 2}\left|a_{n}^{2}-x_{j, n}^{2}\right|^{v / 4} \\
& \times \frac{1}{\left|x-x_{j, n}\right|}\left(1-\frac{\left|x_{j, n}\right|}{a_{n}}\right)^{-v / 2}\left(1-\frac{\left|x_{j, n}\right|}{a_{2 n}}\right)^{v / 2+1} \\
\leqslant & C(f) \varepsilon_{n} \frac{a_{n}}{n} \Phi_{n}^{v / 2}(x) \Phi_{n}^{3 v / 4}\left(x_{j, n}\right)\left(1-\frac{\left|x_{j, n}\right|}{a_{n}}\right)^{-v / 4}\left(1-\frac{\left|x_{j, n}\right|}{a_{2 n}}\right)^{v / 2+1} \frac{1}{\left|x-x_{j, n}\right|} \\
\leqslant & C(f) \varepsilon_{n} \frac{a_{n}}{n}\left(1-\frac{|x|}{a_{n}}\right)^{v / 2}\left(1-\frac{\left|x_{j, n}\right|}{a_{n}}\right)^{v / 2}\left(1-\frac{\left|x_{j, n}\right|}{a_{2 n}}\right)^{v / 2+1} \frac{1}{\left|x-x_{j, n}\right|} .
\end{aligned}
$$

Therefore, since we know from (4.23) that

$$
\frac{1}{\left|x-x_{j, n}\right|} \leqslant C \frac{n / a_{n}}{|m-j|\left(1-\max \left\{|x|,\left|x_{j, n}\right|\right\} / a_{n}\right)^{1 / 2}}
$$

noting (4.24), we have

$$
\begin{aligned}
\sum_{j \neq m} B_{v-1, j}(x) & \leqslant C(f) \varepsilon_{n}\left(1-\frac{|x|}{a_{n}}\right)^{v / 2} \sum_{j \neq m} \frac{\left(1-\left|x_{j, n}\right| / a_{n}\right)^{v / 2}\left(1-\left|x_{j, n}\right| / a_{2 n}\right)^{v / 2+1}}{|m-j|\left(1-\max \left\{|x|,\left|x_{j, n}\right|\right\} / a_{n}\right)^{1 / 2}} \\
& \leqslant C(f) \varepsilon_{n}\left(1-\frac{|x|}{a_{n}}\right)^{(v-1) / 2} \sum_{j \neq m} \frac{\left(1-\left|x_{j, n}\right| / a_{n}\right)^{(v-1) / 2}\left(1-\left|x_{j, n}\right| / a_{2 n}\right)^{v / 2+1}}{|m-j|} \\
& \leqslant C(f) \varepsilon_{n} \sum_{j \neq m} \frac{1}{|m-j|} \leqslant C(f) \varepsilon_{n} \log n .
\end{aligned}
$$

Finally, we estimate $\sum_{j=1}^{n} B_{v-1, j}(x)$ for $x>x_{0, n}$. Suppose $x>x_{0, n}$. Then similar to the above computations, we have

$$
\begin{aligned}
B_{v-1, j}(x) & \leqslant C(f) \varepsilon_{n} \frac{a_{n}}{n} \Phi^{v / 2}(x) \Phi_{n}^{3 v / 4}\left(x_{j, n}\right)\left(1-\frac{\left|x_{j, n}\right|}{a_{n}}\right)^{-v / 4}\left(1-\frac{\left|x_{j, n}\right|}{a_{2 n}}\right)^{v / 2+1} \frac{1}{\left|x_{0, n}-x_{j, n}\right|} \\
& \leqslant C(f) \varepsilon_{n} \frac{a_{n}}{n} \Phi^{v / 2}(x)\left(1-\frac{\left|x_{j, n}\right|}{a_{n}}\right)^{v / 2}\left(1-\frac{\left|x_{j, n}\right|}{a_{2 n}}\right)^{v / 2+1} \frac{1}{\left|x_{0, n}-x_{j, n}\right|} .
\end{aligned}
$$

Then, since we know by (4.28) that

$$
\frac{1}{\left|x_{0, n}-x_{j, n}\right|} \leqslant C \frac{1}{j \delta_{n}^{1 / 2}} \frac{n}{a_{n}}
$$


we have

$$
B_{v-1, j}(x) \leqslant C(f) \varepsilon_{n} \Phi^{v / 2}(x)\left(1-\frac{\left|x_{j, n}\right|}{a_{n}}\right)^{v / 2}\left(1-\frac{\left|x_{j, n}\right|}{a_{2 n}}\right)^{v / 2+1} \frac{1}{j \delta_{n}^{1 / 2}}
$$

Then, since $\Phi^{v / 2}(x) \delta_{n}^{-1 / 2}<C$ for $x>x_{0, n}$, we have

$$
\sum_{j=1}^{n} B_{v-1, j}(x) \leqslant C(f) \varepsilon_{n} \sum_{j=1}^{n} \frac{1}{j} \leqslant C(f) \varepsilon_{n} \log n .
$$

Therefore, the result is proved.

Proof of Proposition 3.9. By Lemma 2.3 we see that, for a constant $C_{1}>0$,

$$
\begin{aligned}
\left|Z_{n}(l, v, f ; x)\right| & \leqslant C_{1} \sum_{j=1}^{n} \sum_{s=1}^{l}\left|f^{(s)}\left(x_{j, n}\right)\right|\left|l_{j n}(x)\right|^{\nu} \sum_{i=s}^{\nu-1}\left(\frac{n}{\sqrt{a_{2 n}^{2}-x_{j, n}^{2}}}\right)^{i-s}\left|x-x_{j, n}\right|^{i} \\
& \leqslant C_{1} \frac{a_{n}}{n} \sum_{j=1}^{n} \sum_{s=1}^{l}\left|f^{(s)}\left(x_{j, n}\right)\right|\left|l_{j n}(x)\right|^{\nu} \sum_{i=s}^{\nu-1}\left(\frac{n}{\sqrt{a_{2 n}^{2}-x_{j, n}^{2}}}\right)\left|x-x_{j, n}\right|^{i} \\
& \leqslant C_{1} \frac{a_{n}}{n} \sum_{s=1}^{l} \sum_{i=0}^{v-1} \sum_{j=1}^{n}\left|f^{(s)}\left(x_{j, n}\right)\right|\left|l_{j n}(x)\right|^{\nu}\left|x-x_{j, n}\right|^{i}\left(\frac{n}{\sqrt{a_{2 n}^{2}-x_{j, n}^{2}}}\right)^{i} \\
& =: \frac{a_{n}}{n} \sum_{s=1}^{l} \sum_{i=0}^{v-1} C_{i, s}(x),
\end{aligned}
$$

where,

$$
C_{i, s}(x):=\sum_{j=1}^{n}\left|f^{(s)}\left(x_{j, n}\right)\right|\left|l_{j n}(x)\right|^{v}\left|x-x_{j, n}\right|^{i}\left(\frac{n}{\sqrt{a_{2 n}^{2}-x_{j, n}^{2}}}\right)^{i}
$$

We set

$$
X_{i, s, n}(v, f ; x):=\sum_{j=1}^{n} f^{(s)}\left(x_{j, n}\right) l_{j n}^{v}(x) e_{i}(v, j, n)\left(x-x_{j, n}\right)^{i}, \quad i=0,1, \ldots, v-1
$$

and hereafter we wrote $(*)$ as $|X|_{i, s, n}(v, f ; x)$. Now, we repeat the proof of Proposition 3.7 by exchanging $f(x)$ with $f^{(s)}(x), s=1,2, \ldots, v-1$, and we note (3.23) (and (3.26)). Then, for $0 \leqslant i \leqslant v-2$ we obtain

$$
\left\{\Phi_{n}^{3 / 4}(x) w(x)\left(|x|+\frac{a_{n}}{n}\right)\right\}^{v}|X|_{i, s, n}(v, f ; x) \leqslant C_{1} C(f) .
$$


For $i=v-1$, we use the estimate for $l_{m n}(x)$ in the proof of Proposition 3.7; furthermore we use Remark 4.4. Then we have

$$
\left\{\Phi_{n}^{3 / 4}(x) w(x)\left(|x|+\frac{a_{n}}{n}\right)\right\}^{v}|X|_{v-1, s, n}(v, f ; x) \leqslant C_{1} C(f) \log n
$$

Consequently, we have

$$
\left\{\Phi_{n}(x)^{3 / 4} w(x)\left(|x|+\frac{a_{n}}{n}\right)\right\}^{v}\left|Z_{n}(l, v, f ; x)\right| \leqslant C_{1} C(f) \frac{a_{n} \log n}{n}
$$

Similarly, we have

$$
\left\{\Phi(x)^{3 / 4} w(x)\left(|x|+\frac{a_{n}}{n}\right)\right\}^{v}\left|W_{n}(l, v, G ; x)\right| \leqslant C_{1} C(G) \frac{a_{n} \log n}{n}
$$

Proof of Proposition 3.10. Let $P \in P_{m}$ be fixed. From Proposition 3.9 we see

$$
\begin{aligned}
& \left\|\left(\Phi^{3 / 4}(x) w(x)\left(|x|+\frac{a_{n}}{n}\right)^{\rho}\right)^{v}\left\{L_{n}(v, P)-P\right\}\right\|_{L_{\infty}(\mathbb{R})} \\
& =\left\|\left(\Phi^{3 / 4}(x) w(x)\left(|x|+\frac{a_{n}}{n}\right)^{\rho}\right)^{v}\left\{L_{n}(v, P)-L_{n}(v-1, v, P)\right\}\right\|_{L_{\infty}(\mathbb{R})} \\
& =\left\|\left(\Phi^{3 / 4}(x) w(x)\left(|x|+\frac{a_{n}}{n}\right)^{\rho}\right)^{v} Z_{n}(v-1, v, P)\right\|_{L_{\infty}(\mathbb{R})} \longrightarrow 0 \quad \text { as } n \longrightarrow \infty .
\end{aligned}
$$

Proof of Proposition 3.11. Let $P \in D_{m}$ with $P(0)=0$. Then $P$ satisfies the condition (A-1). By Proposition 3.8, we see

$$
\left\|\left(\Phi^{3 / 4}(x) w(x)\left(|x|+\frac{a_{n}}{n}\right)^{\rho}\right)^{\nu} Y_{n}(v, P)\right\|_{L_{\infty}(\mathbb{R})} \longrightarrow 0 \quad \text { as } n \longrightarrow \infty
$$

So, from Propositions 3.10 and 3.8 (noting (3.3)) we have

$$
\begin{aligned}
& \left\|\left(\Phi^{3 / 4}(x) w(x)\left(|x|+\frac{a_{n}}{n}\right)^{\rho}\right)^{v}\left(X_{n}(v, P)-P\right)\right\|_{L_{\infty}(\mathbb{R})} \\
& \leqslant C_{1}\left\|\left(\Phi^{3 / 4}(x) w(x)\left(|x|+\frac{a_{n}}{n}\right)^{\rho}\right)^{v}\left(L_{n}(v, P)-P\right)\right\|_{L_{\infty}(\mathbb{R})} \\
& \quad+\left\|\left(\Phi^{3 / 4}(x) w(x)\left(|x|+\frac{a_{n}}{n}\right)^{\rho}\right)^{\rho} Y_{n}(v, P)\right\|_{L_{\infty}(\mathbb{R})} \longrightarrow 0 \quad \text { as } n \longrightarrow \infty
\end{aligned}
$$


Proof of Theorem 3.12. Since $f$ satisfies (3.22), we see

$$
\lim _{|x| \rightarrow \infty}|f(x)| w(x)^{\nu-\delta}=0 .
$$

For a given $\varepsilon>0$, there exists a polynomial $P \in D_{m}$ with $P(0)=0$ such that

$$
\sup _{x \in \mathbb{R}}|f(x)-P(x)|\left\{\Phi^{-3 / 4}(x) w(x)\left(|x|+\frac{a_{n}}{n}\right)^{\rho}\right\}^{\nu}<\varepsilon .
$$

In fact, by [21, Theorem 1.4], there exists a polynomial $R \in D_{m}$ such that

$$
\sup _{x \in \mathbb{R}}|f(x)-R(x)| w^{v-\delta}(x)<\frac{\varepsilon}{2}
$$

Let $P(x):=R(x)-R(0)$. Noting that

$$
\left\{\Phi^{-3 / 4}(x) w(x)\left(|x|+\frac{a_{n}}{n}\right)^{\rho}\right\}^{\nu} \leqslant C w^{\nu-\delta}(x), \quad x \in \mathbb{R} \text { for some } C>0
$$

and $f(0)=0$, we have

$$
\begin{aligned}
& \sup _{x \in \mathbb{R}}|f(x)-P(x)|\left\{\Phi^{-3 / 4}(x) w(x)\left(|x|+\frac{a_{n}}{n}\right)^{\rho}\right\}^{\nu} \\
& \leqslant \sup _{x \in \mathbb{R}}|f(x)-P(x)| w^{v-\delta}(x) \\
& \leqslant \sup _{x \in \mathbb{R}}|f(x)-R(x)| w^{v-\delta}(x)+|f(0)-R(0)| w^{\nu-\delta}(0)<\varepsilon ;
\end{aligned}
$$

that is, we have (4.55). Here, we know that

$$
L_{n}(\nu, f)(x)-f(x)=X_{n}(v, f-P)+\left(X_{n}(v, P)-P\right)+(P-f)+Y_{n}(v, f) .
$$


Therefore, by Propositions 3.7, 3.11, and 3.8, we have for $n$ large enough

$$
\begin{aligned}
&\left\|\left(\Phi^{3 / 4}(x) w(x)\left(|x|+\frac{a_{n}}{n}\right)^{\rho}\right)^{v}\left(L_{n}(v, f)-f\right)\right\|_{L_{\infty}(\mathbb{R})} \\
& \leqslant C\left\|\left(\Phi^{3 / 4}(x) w(x)\left(|x|+\frac{a_{n}}{n}\right)^{\rho}\right)^{v} X_{n}(v, f-P)\right\|_{L_{\infty}(\mathbb{R})} \\
&+\left\|\left(\Phi^{3 / 4}(x) w(x)\left(|x|+\frac{a_{n}}{n}\right)^{\rho}\right)^{v}\left(X_{n}(v, P)-P\right)\right\|_{L_{\infty}(\mathbb{R})} \\
&+\left\|\left(\Phi^{3 / 4}(x) w(x)\left(|x|+\frac{a_{n}}{n}\right)^{\rho}\right)^{v}(P-f)\right\|_{L_{\infty}(\mathbb{R})} \\
&+\left\|\left(\Phi^{3 / 4}(x) w(x)\left(|x|+\frac{a_{n}}{n}\right)^{\rho}\right)^{v} Y_{n}(v, f)\right\|_{L_{\infty}(\mathbb{R})} \\
&=: I_{n}+I I_{n}+I I I_{n}+I V_{n} .
\end{aligned}
$$

Here we see that, by Proposition 3.7 with $\varepsilon:=C(f-P)$ (constant depending only on $f-P$ ) and (4.55),

$$
I_{n} \leqslant C \varepsilon, \quad I I I_{n} \leqslant \varepsilon,
$$

and for $n \geqslant n_{0}$ large enough, we have

$$
\begin{gathered}
I I_{n} \leqslant C \varepsilon, \quad(\text { by Proposition 3.9) } \\
I V_{n} \leqslant C \varepsilon, \quad(\text { by Proposition 3.6). }
\end{gathered}
$$

Consequently, noting (3.3), we have

$$
\lim _{n \rightarrow \infty}\left\{I_{n}+I I_{n}+I I I_{n}+I V_{n}\right\}=0
$$

Then we have Theorem 3.12.

Proof of Theorem 3.13. From Theorem 3.12 and Proposition 3.9, we have

$$
\begin{aligned}
& \left\|\left(\Phi^{3 / 4}(x) w(x)\left(|x|+\frac{a_{n}}{n}\right)^{\rho}\right)^{v}\left(L_{n}(l, v, f)-f\right)\right\|_{L_{\infty}(\mathbb{R})} \\
& \leqslant C_{1}\left\|\left(\Phi^{3 / 4}(x) w(x)\left(|x|+\frac{a_{n}}{n}\right)^{\rho}\right)^{v}\left(L_{n}(v, f)-f\right)\right\|_{L_{\infty}(\mathbb{R})} \\
& \quad+\left\|\left(\Phi^{3 / 4}(x) w(x)\left(|x|+\frac{a_{n}}{n}\right)^{\rho}\right)^{v} Z_{n}(l, v, f)\right\|_{L_{\infty}(\mathbb{R})} \longrightarrow 0 \text { as } n \rightarrow \infty .
\end{aligned}
$$


Proof of Corollary 3.14. From Theorem 3.12 and Proposition 3.8, we have

$$
\begin{aligned}
& \left\|\left(\Phi^{3 / 4}(x) w(x)\left(|x|+\frac{a_{n}}{n}\right)^{\rho}\right)^{v}\left(X_{n}(v, f)-f\right)\right\|_{L_{\infty}(\mathbb{R})} \\
& \leqslant C_{1}\left\|\left(\Phi^{3 / 4}(x) w(x)\left(|x|+\frac{a_{n}}{n}\right)^{\rho}\right)^{v}\left(L_{n}(v, f)-f\right)\right\|_{L_{\infty}(\mathbb{R})} \\
& \quad+\left\|\left(\Phi^{3 / 4}(x) w(x)\left(|x|+\frac{a_{n}}{n}\right)^{\rho}\right)^{v} Y_{n}(v, f)\right\|_{L_{\infty}(\mathbb{R})} \longrightarrow 0 \text { as } n \longrightarrow \infty .
\end{aligned}
$$

Proof of Theorem 3.15. From Theorem 3.13 and Proposition 3.10 we have

$$
\begin{aligned}
&\left\|\left(\Phi^{3 / 4}(x) w(x)\left(|x|+\frac{a_{n}}{n}\right)^{\rho}\right)^{v}\left(\tilde{L}_{n}(l, v, f \oplus G)-f\right)\right\|_{L_{\infty}(\mathbb{R})} \\
& \leqslant C\left\|\left(\Phi^{3 / 4}(x) w(x)\left(|x|+\frac{a_{n}}{n}\right)^{\rho}\right)^{v}\left(L_{n}(l, v, f)-f\right)\right\|_{L_{\infty}(\mathbb{R})} \\
& \quad+\left\|\left(\Phi^{3 / 4}(x) w(x)\left(|x|+\frac{a_{n}}{n}\right)^{\rho}\right)^{\nu} W_{n}(l, v, G)\right\|_{L_{\infty}(\mathbb{R})} \longrightarrow 0 \text { as } n \longrightarrow \infty
\end{aligned}
$$

Proof of Theorem 3.16. We use Theorems 3.12, 3.13, and Corollary 3.14, and Theorem 3.15. Then we have

$$
\begin{aligned}
\left|I_{n}\left[k, H_{n}(f) ; f\right]-I[k, f]\right| & \\
= & \int_{-\infty}^{\infty} k(x)\left\{H_{n}(f)(x)-f(x)\right\} d x \\
\leqslant & C\left\|\left(\Phi^{3 / 4}(x) w(x)\left(|x|+\frac{a_{n}}{n}\right)^{\rho}\right)^{\nu}\left\{H_{n}(f)(x)-f(x)\right\}\right\|_{L_{\infty}(\mathbb{R})} \\
\quad & \times \int_{-\infty}^{\infty}\left(\Phi^{3 / 4}(x) w(x)\left(|x|+\frac{a_{n}}{n}\right)^{\rho}\right)^{v} k(x) d x \longrightarrow 0 \text { as } n \longrightarrow \infty .
\end{aligned}
$$

\section{Divergence Theorem}

If $v$ is a positive odd integer, then we obtain the unboundedness of $L_{n}(v, f ; x)$. We define

$$
\Lambda_{n}(v, \mathbb{R})=\max _{x \in \mathbb{R}} \sum_{k=1}^{n}\left|h_{k n}(x)\right|
$$

Theorem 5.1 (cf. [1, Theorem 2]). Let $v>0$ be an odd integer. Then there exists a constant $C>0$ and $n_{0}>0$ such that for $n \geqslant n_{0}$

$$
\Lambda_{n}(v, \mathbb{R}) \geqslant C \log n
$$


Let $-\infty \leqslant a<b \leqslant \infty$, and let us define

$$
\Lambda_{n}(v,(a, b))=\max _{a \leqslant x \leqslant b} \sum_{k=1}^{n}\left|h_{k n}(x)\right|
$$

Then we will show that for $n \geqslant n_{0}$

$$
\Lambda_{n}(v,(a, b)) \geqslant C \log n
$$

Remark 5.2. For the interpolation polynomial $L_{n}(v, f ; x)$, we can see a remarkable difference between the cases of an odd number $v$ and of an even number $v$. Let us consider any continuous function $f \in C([a, b]), 0<a<b$. Then we can extend $f$ to a continuous function $f^{*} \in C(\mathbb{R})$ which satisfies (3.22) and $f(x)=f^{*}(x), x \in[a, b]$. Then from Theorem 3.12 for an even positive integer $v$, we see

$$
\left\|L_{n}\left(\nu, f^{*} ; x\right)-f(x)\right\|_{L_{\infty}([a, b])} \longrightarrow 0 \quad \text { as } n \longrightarrow \infty \text {. }
$$

On the other hand, the standard argument (cf, [22, Theorem 4.3]) leads us to the following. Theorem 5.1 means that there exists a certain function $f^{*} \in C(\mathbb{R})$ such that

$$
\left\|L_{n}\left(\mathcal{v}, f^{*} ; x\right)-f^{*}(x)\right\|_{L_{\infty}([a, b])} \nrightarrow 0 \quad \text { as } n \longrightarrow \infty ;
$$

that is, for $f(x):=f^{*}(x), a \leqslant x \leqslant b$ we see that the interpolation polynomials $L_{n}\left(v, f^{*} ; x\right)$ do not converge to $f$. We also remark that the polynomial $L_{n}\left(v, f^{*} ; x\right)$ interpolates $f \in C[a, b]$ at only $\left\{x_{j, n} \in[a, b], 1 \leqslant j \leqslant n\right\}$.

To prove the theorem we use the following lemma.

Lemma 5.3 (see [18, Theorem 11]). For $j=0,1,2, \ldots$, there is a polynomial $\Psi_{j}(x)$ of degree $j$ such that $(-1)^{j} \Psi_{j}(-v)>0$ for $v=1,3,5, \ldots$, and the following relation holds. Let $0<\varepsilon<1$. Then one has an expression for $(1 / \varepsilon)\left(a_{n} / n\right) \leqslant\left|x_{k, n}\right| \leqslant \varepsilon a_{n}$ and $s=0,1, \ldots,(v-1) / 2$ :

$$
e_{2 s}(v, k, n)=(-1)^{s}\left\{\frac{1}{(2 s) !}\right\} \Psi_{s}(-v) \beta^{s}(n, k)\left(\frac{n}{a_{n}}\right)^{2 s}\left\{1+\eta_{k n}(v, s)\right\}
$$

where for $\beta^{s}(n, k), n=1,2,3, \ldots, k=1,2, \ldots, n, s=0,1, \ldots,(v-1) / 2$, there exist the constants $C_{1}, C_{2}$ such that

$$
0<C_{1} \leqslant \beta^{s}(n, k) \leqslant C_{2}
$$


and $\eta_{k n}(v, s)$ satisfies

$$
\left|\eta_{k n}(\mathcal{v}, s)\right| \leqslant \varepsilon_{n^{\prime}}^{*} \quad \varepsilon_{n}^{*}=\varepsilon_{n}^{*}(\varepsilon) \longrightarrow 0 \quad \text { as } \varepsilon \longrightarrow 0,
$$

for $k$ with $(1 / \varepsilon)\left(a_{n} / n\right) \leqslant\left|x_{k, n}\right| \leqslant \varepsilon a_{n}$, and for $s=0,1,2, \ldots$

Proof of Theorem 5.1. To get a lower bound of $\Lambda_{n}(v, \mathbb{R})$, it suffices to consider a lower bound (5.4) of $\Lambda_{n}(v,[a, b])$ with $0<a<b<\infty$. Let $c:=a / 2, d:=2 b-a$ and we consider the intervals $I:=[a, b], J=[c, d]$. If we consider $n$ large enough, then we have $(1 / \varepsilon)\left(a_{n} / n\right) \leqslant c<2 b-a \leqslant$ $\varepsilon a_{n}$. See the expression

$$
\left|h_{k n}(x)\right| \geqslant\left|l_{k n}^{v}(x) e_{v-1}(v, k, n)\left(x-x_{k, n}\right)^{v-1}\right|-\left|l_{k n}^{v}(x) \sum_{i=0}^{v-2} e_{i}(v, k, n)\left(x-x_{k, n}\right)^{i}\right| .
$$

Then we have

$$
\begin{aligned}
\Lambda_{n}(v,[a, b]) \geqslant & \max _{x \in I} \sum_{x_{k n} \in J}\left|l_{k n}^{v}(x) e_{v-1}(v, k, n)\left(x-x_{k, n}\right)^{v-1}\right| \\
& -\max _{x \in I} \sum_{x_{k n} \in J}\left|l_{k n}^{v}(x) \sum_{i=0}^{v-2} e_{i}(v, k, n)\left(x-x_{k, n}\right)^{i}\right| \\
= & \max _{x \in I} F_{n}(x)-\max _{x \in I} G_{n}(x) .
\end{aligned}
$$

It follows from Remark 4.5 that $\max _{a \leqslant x \leqslant b} G_{n}(x) \leqslant C$. Hence, it is enough to show that $\max _{x \in I} F_{n}(x) \geqslant C \log n$. Let $x_{k+1, n}, x_{k, n} \in J$; then by Lemma 4.2 and the definition of $\varphi_{n}\left(x_{k, n}\right)$ there exists $0<\alpha \leqslant \beta<\infty$ such that

$$
\alpha \frac{a_{n}}{n} \leqslant\left|x_{k, n}-x_{k+1, n}\right| \leqslant \beta \frac{a_{n}}{n} .
$$

For $x \in I$ we consider only $x_{k, n} \in J$ such that, for some positive integer $j_{k}$,

$$
\left(j_{k}-1\right) \alpha \frac{a_{n}}{n} \leqslant\left|x_{k, n}-x\right| \leqslant j_{k} \beta \frac{a_{n}}{n} .
$$

Then for each $x \in I$ we define

$$
\Gamma(x):=\left\{j_{k} ; x_{k, n} \in J,\left(j_{k}-1\right) \alpha \frac{a_{n}}{n} \leqslant\left|x_{k, n}-x\right| \leqslant j_{k} \beta \frac{a_{n}}{n}\right\} .
$$


Here, we will see that

$$
\left\{j ; 1 \leqslant j \leqslant \frac{d-c}{2 \beta} \frac{n}{a_{n}}-1\right\} \subset \Gamma(x) .
$$

Let

$$
\begin{aligned}
& x_{m+1, n}<x \leqslant x_{m, n} \\
& x_{k(c)+1, n}<c \leqslant x_{k(c), n}, x_{k(d), n} \leqslant d<x_{k(d)-1, n} .
\end{aligned}
$$

Then we have

$$
\begin{gathered}
x-x_{m+1, n} \leqslant x_{m, n}-x_{m+1, n} \leqslant \beta \frac{a_{n}}{n}, \\
x-x_{m+2, n} \leqslant x_{m, n}-x_{m+2, n} \leqslant 2 \beta \frac{a_{n}}{n}, \\
\vdots \\
x-x_{k(c), n} \leqslant x_{m, n}-x_{k(c), n} \leqslant j(c) \beta \frac{a_{n}}{n}, \\
x_{m, n}-x \leqslant x_{m, n}-x_{m+1, n} \leqslant \beta \frac{a_{n}}{n}, \\
x_{m-1, n}-x \leqslant x_{m-1, n}-x_{m+1, n} \leqslant 2 \beta \frac{a_{n}}{n}, \\
\vdots \\
x_{k(d), n}-x \leqslant x_{k(d), n}-x_{m+1, n} \leqslant j(d) \beta \frac{a_{n}}{n},
\end{gathered}
$$

where $j(c)$ and $j(d)$ are integers. On the other hand,

$$
\frac{\left(x_{k(d), n}-x_{k(c), n}\right)}{\left(\beta a_{n} / n\right)} \geqslant \frac{\left(d-c-2 \beta a_{n} / n\right)}{\left(\beta a_{n} / n\right)}=\frac{d-c}{\beta} \frac{n}{a_{n}}-2 .
$$

Therefore, we have

$$
\max (j(c), j(d)) \geqslant \frac{1}{2}\left(\frac{d-c}{\beta} \frac{n}{a_{n}}-2\right)=\frac{d-c}{2 \beta} \frac{n}{a_{n}}-1 .
$$

Now, we take a positive integer $N(n)$ such that

$$
N(n) \leqslant \frac{d-c}{2 \beta} \frac{n}{a_{n}}-1<N(n)+1 .
$$


Consequently, we have the following. By Lemmas 4.2(1), Lemma 4.1(1), (2), (4), and Lemma 2.3, we have

$$
\begin{aligned}
F_{n}(x) & =\sum_{x_{k, n} \in J}\left|l_{k n}(x)\right|^{v}\left|e_{v-1}(v, k, n)\right|\left|x_{k, n}-x\right|^{\nu-1} \\
& =\sum_{x_{k, n} \in J}\left(\frac{\left|p_{n}(x)\right| w_{\rho}(x)}{\left|x-x_{k, n}\right|\left|p_{n}^{\prime}\left(x_{k, n}\right)\right| w_{\rho}\left(x_{k, n}\right)} \frac{w_{\rho}\left(x_{k, n}\right)}{w_{\rho}(x)}\right)^{v}\left|e_{v-1}(v, k, n)\right|\left|x-x_{k, n}\right|^{\nu-1} \\
& =\sum_{x_{k, n} \in J}\left(\frac{\left|p_{n}(x)\right| w_{\rho}(x)}{\left|p_{n}^{\prime}\left(x_{k, n}\right)\right| w_{\rho}\left(x_{k, n}\right)} \frac{w_{\rho}\left(x_{k, n}\right)}{w_{\rho}(x)}\right)^{v}\left|e_{v-1}(v, k, n)\right| \frac{1}{\left|x-x_{k, n}\right|} \\
& \geqslant C \sum_{j_{k} \in \Gamma(x)}\left(\frac{a_{n}^{-1 / 2}}{\left(n / a_{n}\right) a_{n}^{-1 / 2} \frac{w_{\rho}(d)}{w_{\rho}(a)}}\right)^{v}\left|e_{v-1}(v, k, n)\right| \frac{n}{j_{k} \beta a_{n}} \\
& \geqslant C \sum_{j_{k} \in \Gamma(x)} \frac{1}{j_{k}}\left(\frac{a_{n}}{n}\right)^{v-1}\left|e_{v-1}(v, k, n)\right| .
\end{aligned}
$$

Here, using Lemma 5.3 and (5.15), we have

$$
F_{n}(x) \geqslant C \sum_{j_{k} \in \Gamma(x)} \frac{1}{j_{k}}\left(\frac{a_{n}}{n}\right)^{\nu-1}\left(\frac{n}{a_{n}}\right)^{\nu-1} \geqslant C \sum_{1 \leqslant j \leqslant N(n)} \frac{1}{j} .
$$

Here, there exists $0<\eta<1$ such that $n^{\eta} \leqslant N(n)$. Therefore we see

$$
F_{n}(x) \geqslant C \sum_{1 \leqslant j \leqslant n^{\eta}} \frac{1}{j} \geqslant C \log n
$$

So we have (5.4), and consequently the proof of Theorem 5.1 is completed.

\section{Acknowledgment}

The authors thank the referees for many kind suggestions and comments.

\section{References}

[1] Y. Kanjin and R. Sakai, "Pointwise convergence of Hermite-Fejér interpolation of higher order for Freud weights," The Tohoku Mathematical Journal, vol. 46, no. 2, pp. 181-206, 1994.

[2] Y. Kanjin and R. Sakai, "Convergence of the derivatives of Hermite-Fejer interpolation polynomials of higher order based at the zeros of Freud polynomials," Journal of Approximation Theory, vol. 80, no. 3, pp. 378-389, 1995.

[3] D. S. Lubinsky, "Hermite and Hermite-Fejér interpolation and associated product integration rules on the real line: the L1 theory," Journal of Approximation Theory, vol. 70, no. 3, pp. 284-334, 1992.

[4] T. Kasuga and R. Sakai, "Uniform or mean convergence of Hermite-Fejér interpolation of higher order for Freud weights," Journal of Approximation Theory, vol. 101, no. 2, pp. 330-358, 1999.

[5] T. Kasuga and R. Sakai, "Orthonormal polynomials with generalized Freud-type weights," Journal of Approximation Theory, vol. 121, no. 1, pp. 13-53, 2003. 
[6] T. Kasuga and R. Sakai, "Orthonormal polynomials for generalized Freud-type weights and higherorder Hermite-Fejér interpolation polynomials," Journal of Approximation Theory, vol. 127, no. 1, pp. 1-38, 2004.

[7] T. Kasuga and R. Sakai, "Orthonormal polynomials for Laguerre-type weights," Far East Journal of Mathematical Sciences, vol. 15, no. 1, pp. 95-105, 2004.

[8] T. Kasuga and R. Sakai, "Conditions for uniform or mean convergence of higher order HermiteFejér interpolation polynomials with generalized Freud-type weights," Far East Journal of Mathematical Sciences, vol. 19, no. 2, pp. 145-199, 2005.

[9] A. L. Levin and D. S. Lubinsky, Orthogonal Polynomials for Exponential Weights, Springer, New York, NY, USA, 2001.

[10] H. S. Jung and R. Sakai, "Specific examples of exponential weights," Korean Mathematical Society, vol. 24, no. 2, pp. 303-319, 2009.

[11] H. S. Jung and R. Sakai, "The Markov-Bernstein inequality and Hermite-Fejér interpolation for exponential-type weights," Journal of Approximation Theory, vol. 162, no. 7, pp. 1381-1397, 2010.

[12] R. Sakai, "Hermite-Fejér interpolation," in Approximation Theory, vol. 58, pp. 591-601, North-Holland, Amsterdam, The Netherlands, 1990.

[13] R. Sakai, "The degree of approximation of differentiable functions by Hermite interpolation polynomials," Acta Mathematica Hungarica, vol. 58, no. 1-2, pp. 9-11, 1991.

[14] R. Sakai, "Hermite-Fejér interpolation prescribing higher order derivatives," in Progress in Approximation Theory, pp. 731-759, Academic Press, Boston, Mass, USA, 1991.

[15] R. Sakai, "Certain bounded Hermite-Fejer interpolation polynomials operator," Acta Mathematica Hungarica, vol. 59, pp. 111-114, 1992.

[16] R. Sakai and P. Vértesi, "Hermite-Fejér interpolations of higher order. III," Studia Scientiarum Mathematicarum Hungarica, vol. 28, no. 1-2, pp. 87-97, 1993.

[17] R. Sakai and P. Vértesi, "Hermite-Fejér interpolations of higher order. IV," Studia Scientiarum Mathematicarum Hungarica, vol. 28, no. 3-4, pp. 379-386, 1993.

[18] H. S. Jung and R. Sakai, "Derivatives of orthonormal polynomials and coefficients of Hermite-Fejér interpolation polynomials with exponential-type weights," Journal of Inequalities and Applications, vol. 2010, Article ID 816363, 29 pages, 2010.

[19] H. S. Jung and R. Sakai, "Orthonormal polynomials with exponential-type weights," Journal of Approximation Theory, vol. 152, no. 2, pp. 215-238, 2008.

[20] H. S. Jung and R. Sakai, "Derivatives of integrating functions for orthonormal polynomials with exponential-type weights," Journal of Inequalities and Applications, vol. 2009, Article ID 528454, 22 pages, 2009.

[21] D. S. Lubinsky, "A survey of weighted polynomial approximation with exponential weights," Surveys in Approximation Theory, vol. 3, pp. 1-105, 2007.

[22] T. J. Rivlin, An Introduction to the Approximation of Functions, Dover, New York, NY, USA, 1981. 


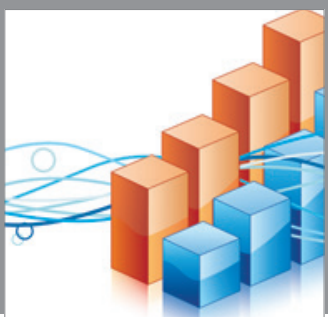

Advances in

Operations Research

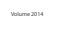

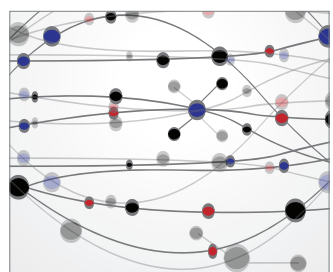

\section{The Scientific} World Journal
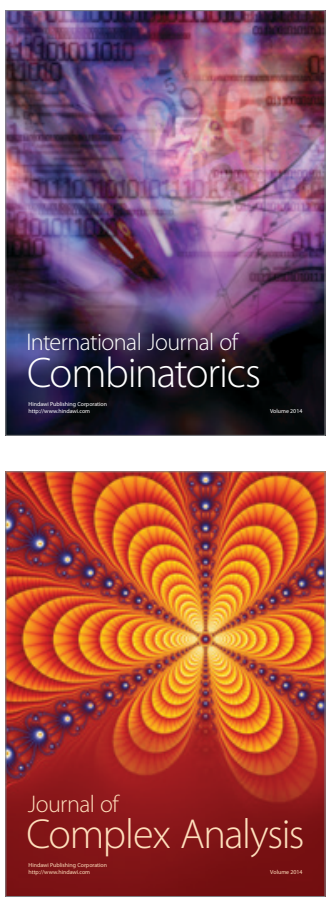

International Journal of

Mathematics and

Mathematical

Sciences
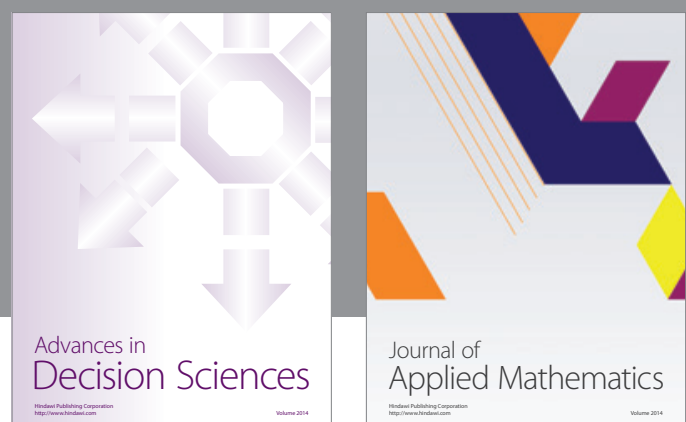

Journal of

Applied Mathematics
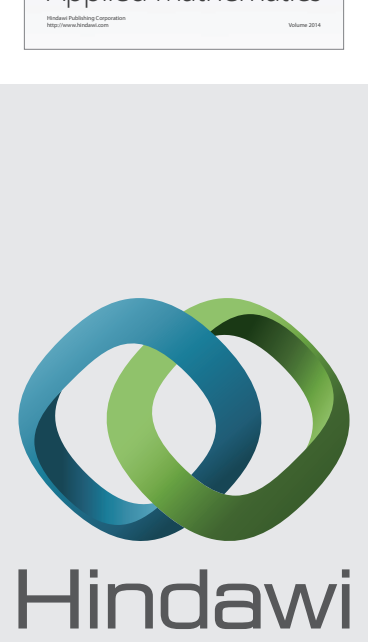

Submit your manuscripts at http://www.hindawi.com
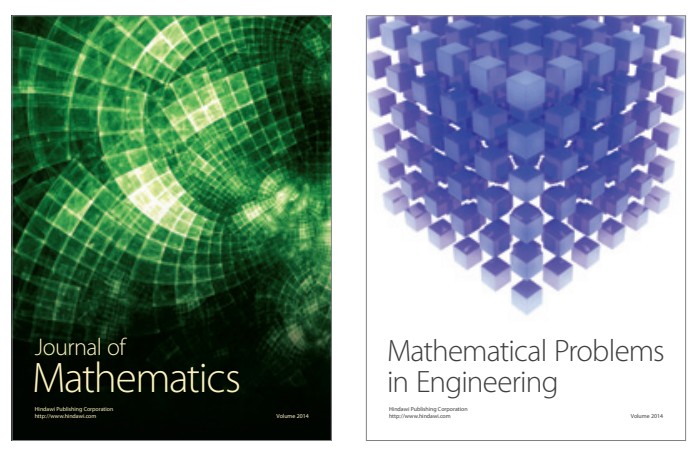

Mathematical Problems in Engineering
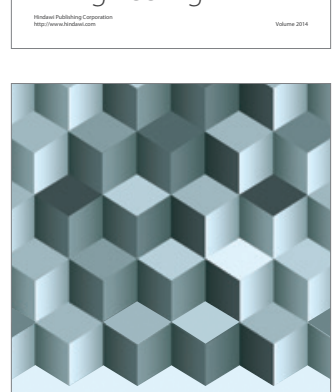

Journal of

Function Spaces
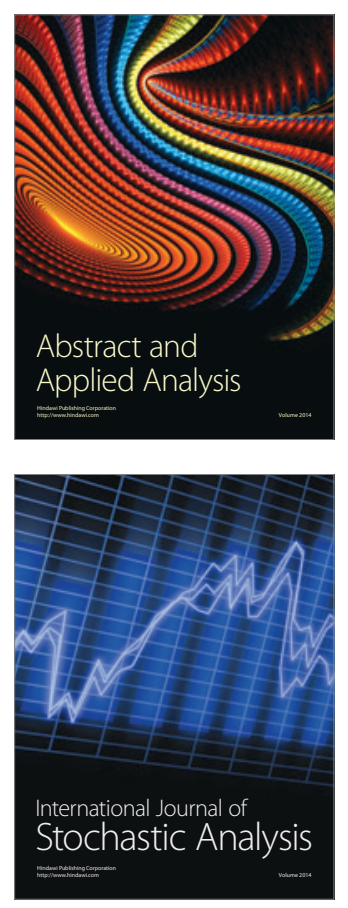

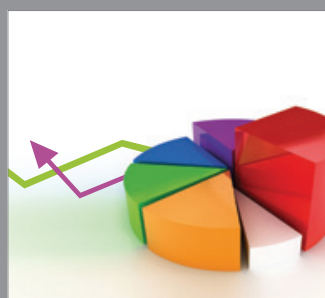

ournal of

Probability and Statistics

Promensencen
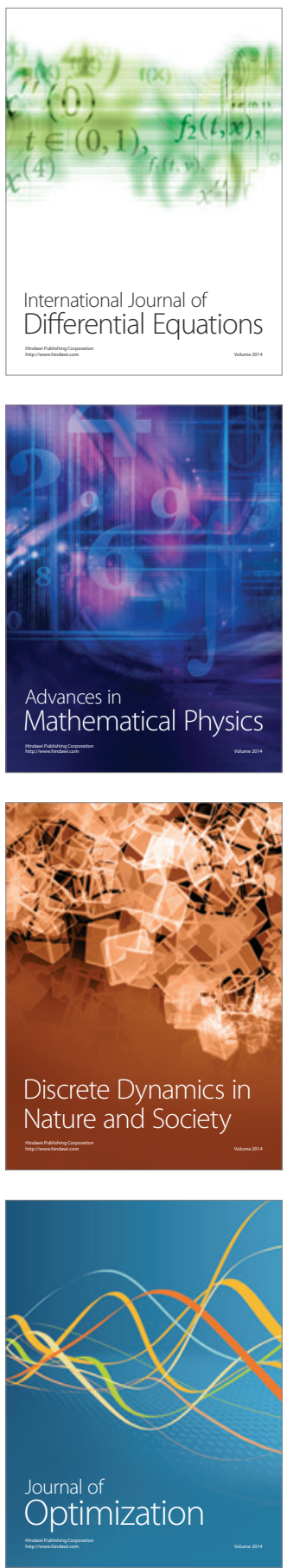